\title{
Boundary Trace of Positive Solutions of Nonlinear Elliptic Inequalities
}

\author{
MOSHE MARCUS - LAURENT VÉRON
}

\begin{abstract}
We develop a new method for proving the existence of a boundary trace, in the class of Borel measures, of nonnegative solutions of $-\Delta u+g(x, u) \geq 0$ in a smooth domain $\Omega$ under very general assumptions on $g$. This new definition which extends the previous notions of boundary trace is based upon a sweeping technique by solutions of Dirichlet problems with measure boundary data. We also prove a boundary pointwise blow-up estimate of any solution of such inequalities in terms of the Poisson kernel. If the nonlinearity is very degenerate near the boundary, for example if $g(x, u) \approx \exp \left(-\rho_{\partial \Omega}^{-1}(x)\right) u^{q}$, we exhibit a new full boundary blow-up phenomenon.
\end{abstract}

Mathematics Subject Classification (2000): 35K60.

\section{Introduction}

Let $\Omega$ be a bounded open domain in $\mathbb{R}^{N}$ with a $C^{2}$ boundary $\partial \Omega$. This paper is concerned with the study of the generalized boundary value problem for the equation

$$
-\Delta u+g(x, u)=0 \text { in } \Omega
$$

where $(x, r) \mapsto g(x, r)$ is a continuous function defined on $\Omega \times \mathbb{R}$, nondecreasing in the $r$ variable, and nonnegative if $r \geq 0$. When $g(r)=r^{q}$ this problem has been thoroughly investigated with a probabilistic approach by Le Gall [19], [20] in the case $N=2=q$, then by Marcus and Veron [21], [22], [23] in the general case $q>1, N>1$ by analytic tools. Related studies were carried on by Dynkin and Kuznetsov [10], [11] with a mixing of probabilistic and analytic methods. In [16] the same problem is investigated with $g(r)=\exp (r)$. In all those cases, the boundary trace dichotomy argument is settled upon duality techniques which were first introduced by Baras and Pierre [1], but in the case of general nonlinearity, this method fails. 
In [27] an new approach of the boundary trace is developed for positive solutions of (0.1). This approach is settled upon two ingredients:

I - The coerciveness, which asserts that the set of nonnegative solutions of $(0.1)$ is bounded in the local uniform topology upon $C(\Omega)$.

II - The strong-barrier property which is the property that for any boundary point $z$ and for any $r>0$, small enough, there exist supersolutions $\varphi$ of (0.1) in $\Omega \cap B_{r}(z)$ with infinite value on $\partial B_{r}(z) \cap \Omega$, and zero value on $\partial \Omega \cap B_{r}(z)$.

When $g$ depends only of $r$, those two notions coincide thanks to the OssermanKeller condition,

$$
\int_{a}^{\infty} \frac{d s}{\sqrt{G(s)}}<\infty, \quad \text { for any } a>0
$$

where $G(s)=\int_{0}^{s} g(t) d t$. The same equivalence holds if $\inf _{x \in \Omega} g(x, r)=g(r)$, and $g$ satisfies (0.2). Moreover, in these cases, the local uniform upper bound of any positive solution of (0.1) achieves the following form

$$
u(x) \leq \psi_{g}\left(\rho_{\partial \Omega}(x)\right), \quad \forall x \in \Omega,
$$

where $\psi_{g}(t)=\int_{t}^{\infty} \frac{d s}{\sqrt{2 G(s)}}$, and $\left.\rho_{\partial \Omega}(x)\right)=\operatorname{dist}(x, \partial \Omega)$.

If the strong barrier property is uniform with respect to $z \in \partial \Omega$, it implies the coerciveness, but when $\lim _{\left.\rho_{\partial \Omega}(x)\right) \rightarrow 0} g(x, r)=0$ for any $r>0$, (we say that the nonlinearity degenerates near the boundary), the reverse implication may not hold. However, if

$$
g(x, r) \geq \rho_{\partial \Omega}^{\alpha}(x) r^{q} \quad \forall(x, r) \in \Omega \times \mathbb{R}_{+},
$$

for some $\alpha>0$ and $q>1$, it is proved in [27] that the equivalence still holds.

We adopt here a different point of view in connecting the existence of a boundary trace and the question of solving a Dirichlet problem with measure data. If $\mu \in \mathfrak{M}(\partial \Omega)$, the set of Radon measures on $\partial \Omega$, and $(x, r) \mapsto g(x, r)$ is a continuous function defined on $\Omega \times \mathbb{R}$, a function $u$ defined in $\Omega$ is a solution of

$$
\begin{aligned}
-\Delta u+g(x, u) & =0 \text { in } \Omega, \\
u & =\mu \text { on } \partial \Omega,
\end{aligned}
$$

if $u \in L^{1}(\Omega), g(., u) \in L^{1}\left(\Omega, \rho_{\partial \Omega} d x\right)$ and

$$
\int_{\Omega}(-u \Delta \zeta+g(x, u) \zeta) d x=-\int_{\partial \Omega} \frac{\partial \zeta}{\partial \mathbf{n}} d \mu(y)
$$


for any $\zeta \in C_{c}^{1,1}(\bar{\Omega})$, the subspace of $C^{1}(\bar{\Omega})$ functions with Lipschitz continuous gradient and zero value on $\partial \Omega$. If $g(., x)$ is nondecreasing, this solution is unique whenever it exists and we denote it by $u=u_{\mu}$, since

$$
\left\|u_{\mu}-u_{\mu^{*}}\right\|_{L^{1}(\Omega)}+\left\|\rho_{\partial \Omega}\left(g\left(u_{\mu}, .\right)-g\left(u_{\mu^{*}}, .\right)\right)\right\|_{L^{1}(\Omega)} \leq C\left\|\mu-\mu^{*}\right\|_{\mathfrak{M}(\partial \Omega)} .
$$

The mapping $\mu \mapsto u_{\mu}$ is nondecreasing, moreover if $g(x, 0)=0, \mu \geq 0 \Longrightarrow$ $u_{\mu} \geq 0$. Conditions for existence are various.

Let $\mathcal{G}_{0}$ be the set of continuous functions $g$ defined in $\Omega \times \mathbb{R}$ such that $g(x, 0)=0$ and $r \mapsto g(r, x)$ is nondecreasing for any $x \in \Omega$, and $(x, y) \mapsto$ $P(x, y)$ be the Poisson kernel in $\Omega \times \partial \Omega$. If $\mu \in \mathfrak{M}(\partial \Omega)$, we denote by $\mathbb{P}_{\mu}$ its Poisson's potential. If $g \in \mathcal{G}_{0}$ we say that $\mu$ is $g$-admissible if

$$
\int_{\Omega} g\left(x, \mathbb{P}_{|\mu|}(x)\right) \rho_{\partial \Omega}(x)<\infty .
$$

It is proved in [27] that problem (0.4) is uniquely solvable if $\mu$ is $g$-admissible.

However to check this condition on every measure might be far out of reach and a more tractable condition is introduced. We denote $\mathcal{H} \mathcal{G}_{0}$ the subset of $g \in \mathcal{G}_{0}$ such that there exist two continuous, nondecreasing and nonnegative functions $h$ and $f$ defined on $\mathbb{R}_{+}$, such that

$$
\begin{aligned}
& 0 \leq|g(x, r)| \leq h\left(\rho_{\partial \Omega}(x)\right) f(|r|), \quad \forall(x, r) \in \Omega \times \mathbb{R}, \\
& \int_{0}^{1} h(s) f\left(\sigma s^{1-N}\right) s^{N} d s<\infty, \quad \forall \sigma \geq 0, \\
& \text { either } h(s)=s^{\alpha} \text {, for some } \alpha \geq 0 \text {, or } f \text { is convex . }
\end{aligned}
$$

In the first section of this article we prove the following.

If $g \in \mathcal{H} \mathcal{G}_{0}$, then for any $\mu \in \mathfrak{M}(\partial \Omega)$, problem (0.4) admits a unique solution $u_{\mu}$. Moreover the problem is stable, in the sense that if $\left\{\mu_{n}\right\} \subset \mathfrak{M}(\partial \Omega)$ converges to $\mu$ in the weak sense of measures on $\partial \Omega$, the corresponding solutions $\left\{u_{\mu_{n}}\right\}$ converge to $u_{\mu}$, locally uniformly in $\Omega$.

In the second section we introduce a new definition of the boundary trace for nonnegative solutions of elliptic inequalities.

$$
-\Delta u+g(x, u) \geq 0 \quad \text { in } \Omega,
$$

which extends the previous results concerning equations. A key observation for defining this notion is a supremum technique introduced by Richard and Véron [30] in the study of isolated singularities of elliptic inequalities. Following [27] we say that a function $g \in \mathcal{G}_{0}$ is positively subcritical if for any $\mu \in \mathfrak{M}_{+}(\partial \Omega)$, problem (0.4) admits a solution $u_{\mu}$ (unique and nonnegative). If $u \in C(\Omega)$ such that $\Delta u \in L_{\mathrm{loc}}^{1}(\Omega)$ is a nonnegative solution of $(0.9)$, then $w_{\mu}=\min \left\{u, u_{\mu}\right\}$ satisfies (0.9), and it admits a boundary trace $\gamma_{u}(\mu) \in \mathfrak{M}_{+}(\partial \Omega)$. Furthermore 
$\mu \mapsto \gamma_{u}(\mu)$ is nondecreasing, concave if $r \mapsto g(x, r)$ is convex. Therefore the formula

$$
v=\sup _{\mu \in \mathfrak{M}_{+}(\partial \Omega)} \gamma_{u}(\mu)
$$

defines a Borel measure $v=\operatorname{Tr}_{\partial \Omega}^{e}(u)$ on $\partial \Omega$, that we call the extended boundary trace of $u$. This measure may not be an outer regular one except in some particular cases. A particularly important case deals with the choice $\mu=\lambda \delta_{a}$ for $\lambda>0, a \in \partial \Omega$. The corresponding solution $u_{\lambda \delta_{a}}$ is called a fundamental solution. In such a case the boundary trace of $w_{\lambda \delta_{a}}$ is a measure concentrated at $a$, that we denote $\tilde{\gamma}_{u}(a, \lambda) \delta_{a}$. The mapping $\lambda \mapsto \tilde{\gamma}_{u}(a, \lambda)$ is a nondecreasing on $\mathbb{R}_{+}$, and satisfies

$$
0 \leq \tilde{\gamma}_{u}(a, \lambda) \leq \lambda, \quad \forall \lambda \geq 0, \forall a \in \partial \Omega .
$$

We define

$$
\tilde{\gamma}_{u}(a)=\lim _{\lambda \rightarrow \infty} \tilde{\gamma}_{u}(a, \lambda)
$$

and denote by $\mathcal{A}(u)$ the set of atoms of $u$,

$$
\mathcal{A}(u)=\left\{a \in \partial \Omega: \gamma_{u}(a)>0\right\} .
$$

The regular set $\mathcal{R}(u)$ of $u$ is the relatively open subset of the boundary points $a$ with the property that there exists a relatively neighborhood of $a, \mathcal{O} \subset \partial \Omega$ such that

$$
\sum_{\omega \in \mathcal{O}} \tilde{\gamma}_{u}(\omega)<\infty
$$

The singular set $\mathcal{S}(u)$ of $u$ is the closed subset of the boundary points $a$ with the property that for any relatively open neighborhood $\mathcal{O} \subset \partial \Omega$ of $a$, there holds

$$
\sum_{\omega \in \mathcal{O}} \tilde{\gamma}_{u}(\omega)=\infty
$$

Those two definitions extend the classical notions of regular or singular sets of the boundary trace of the solution of an equation (see [22], [24]).

We prove in particular

$$
v(a)=\tilde{\gamma}_{u}(a), \quad \forall a \in \partial \Omega,
$$

and the equivalence between

(i) $v(\mathcal{O})=\infty$, for any relatively open neighborhood $\mathcal{O} \subset \partial \Omega$ of $a$, and

(ii) $u_{\infty, a} \leq u$,

under a general stability condition which holds in particular if $g \in \mathcal{H} \mathcal{G}_{0}$. 
As for $u_{\infty, a}$, different features may occur, in particular,

- $u_{\infty, a} \equiv+\infty$, the full blow-up case.

- $u_{\infty, a}(x)<\infty$ for any $x \in \Omega$, but $\lim _{\rho_{\partial \Omega}(x) \rightarrow 0} u_{\infty, a}(x)=\infty$, the uniform boundary blow-up case.

- $u_{\infty, a}(x)<\infty$ for any $x \in \bar{\Omega} \backslash\{a\}$, and $\lim _{x \rightarrow a} u_{\infty, a}(x)=\infty$ (non-tangential limit), the strong isolated singularity case.

Using precise pointwise estimates of positive super-harmonic functions near the boundary and the sweeping of any positive solution $u$ of (0.9) by the solutions of (0.4) with Dirac masses as boundary data, we prove that for any $a \in \partial \Omega$,

$$
x \mapsto|x-a|^{N-1} u(x)
$$

converges in measure on the set $\{\sigma=(y-a) /|y-a|: y \in \Omega\}$ to $C(N) \tilde{\gamma}_{u}(a)$ as $x \rightarrow a$, where $C(N)$ is some positive constant depending only on the dimension.

If $g(x, r)$ satisfies

$$
g(x, r) \geq \tilde{h}(x) \tilde{g}(r), \forall(x, r) \in \Omega \times \mathbb{R}_{+},
$$

where $\tilde{h} \in C(\Omega)$ takes positive values, and $\tilde{g}$ is nondecreasing and satisfies $(0.2)$, there exists a maximal solution $U_{M}$ to $(0.1)$ in $\Omega$ (actually the global positivity of $\tilde{h}$ can be weakened, since the positivity near $\partial \Omega$ is sufficient for the existence of $U_{M}$ ). In that case (ii) implies

$$
u_{\infty, a}(x) \leq u(x) \leq U_{M}(x),
$$

which rules out the full blow-up case. The nature of $u_{\infty, a}$ depends strongly on $\tilde{h}$ and $\tilde{g}$. For example if it is assumed that $\tilde{h}$ is a positive constant, it follows from the method of construction of maximal solutions that the uniform boundary blow-up case does not hold, and we are left with the strong isolated singularity case. However, this situation also holds even if $\tilde{h}$ depends truly of $x$. It is proved in [27] that if

(0.12) $h(x)=\rho_{\partial \Omega}^{\alpha}(x)$, with $\alpha>-2$ and $1<q<(N+\alpha+1) /(N-1)=q_{c}(\alpha)$, the strong isolated singularity case occurs. We prove here that if

$$
g(x, r)=\exp \left(-1 / \rho_{\partial \Omega}(x)\right) r^{q}, \quad \text { with } q>1,
$$

the uniform boundary blow-up case occurs for any $a \in \partial \Omega$. In such a case, either the boundary trace is a bounded Borel measure, or $u \equiv U_{M}$.

A parabolic version of this phenomenon has been observed in [28]. 
When the nonlinearity is not degenerate in the sense that the function $g \in \mathcal{H} \mathcal{G}_{0}$ satisfies

$$
0 \leq|g(x, r)| \leq f(|r|), \forall(x, r) \in \Omega \times \mathbb{R}, \text { and } \int_{0}^{1} f\left(s^{1-N}\right) s^{N} d s<\infty,
$$

where $f$ is a continuous nondecreasing function defined on $\mathbb{R}_{+}$, we recover the classical definition of the boundary trace in the class of outer regular Borel measures. More precisely, if $u$ is a nonnegative solution of (0.9) with extended boundary trace $\nu$, then for any point $a \in \partial \Omega$ the following dichotomy occurs: either

(i) $a \in \mathcal{S}(u)$ and for any $\mathcal{O} \in \mathcal{N}_{a}$ (the set of its relatively open neighborhoods $\mathcal{O} \subset \partial \Omega), v(\mathcal{O})=\infty$. This is equivalent to

$$
\lim _{t \rightarrow 0} \int_{\mathcal{O}_{t}} u(y) d S_{t}=\infty, \forall \mathcal{O} \in \mathcal{N}_{a},
$$

where $\mathcal{O}_{t}$ is the subset of points in $\Omega$ at distance $t>0$ from $\partial \Omega$, with projection in $\mathcal{O}$ and $d S_{t}$ the induced $(N-1)$-dimensional Hausdorff measure, or

(ii) $a \in \mathcal{R}(u)$, there exists $\mathcal{O} \in \mathcal{N}_{a}$ such that $v(\mathcal{O})<\infty$ and for any $\mathcal{O}^{\prime} \subset$ $\overline{\mathcal{O}}^{\prime} \subset \mathcal{O}$

$$
\sup _{t \in\left(0, \beta_{0}\right]} \int_{\mathcal{O}_{t}} u(y) d S<\infty .
$$

Furthermore, for any $\phi \in C_{c}(\mathcal{R}(u))$

$$
\lim _{t \rightarrow 0} \int_{\mathcal{O}_{t}} u(y) \phi d S_{t}=\int_{\mathcal{R}(u)} \phi d v .
$$

Our paper is organised as follows: In Section 1 we study the boundary value problem with Radon measures. In Section 2 we define and study the extended boundary trace of nonnegative solutions of inequalities. In Section 3 we give a boundary pointwise estimate for solutions of inequalities. In Section 4 we give properties of the boundary trace when the nonlinearity is not degenerate at the boundary. In Section 5 we study different examples of limit of a fundamental solution when the mass goes to infinity.

\section{1. - Measure boundary data}

Throughout this section, $\Omega$ is a bounded domain with a $C^{2}$ boundary $\partial \Omega$ and $\rho_{\partial \Omega}(x)=\operatorname{dist}(x, \partial \Omega)$. We put

(1.1) $\mathcal{G}_{0}=\{g \in C(\Omega \times \mathbb{R})$ s.t. $g(x, 0)=0$ and $r \mapsto g(x, r)$ nondecreasing, $\forall x \in \Omega\}$. 
We denote by $C_{c}^{1,1}(\bar{\Omega})$, the subspace of $C^{1}(\bar{\Omega})$-functions with Lipschitz continuous gradient and zero value on $\partial \Omega, \mathfrak{M}(\partial \Omega)$ the space of Radon measures on $\partial \Omega$, and $\mathfrak{M}_{+}(\partial \Omega)$ its positive cone. If $P(x, y)$ is the Poisson kernel in $\Omega \times \partial \Omega$, the Poisson potential of $\mu$ denoted by $\mathbb{P}_{\mu}$ is defined by

$$
\mathbb{P}_{\mu}(x)=\int_{\partial \Omega} P(x, y) d \mu(y), \quad \forall x \in \Omega .
$$

The next variant of Herglotz' theorem is due to Brezis [5] (see [32] for a proof).

LeMmA 1.1. Let $f \in L^{1}\left(\Omega ; \rho_{\partial \Omega} d x\right)$ and $\varphi \in L^{1}(\partial \Omega)$. Then there exists a unique $u \in L^{1}(\Omega)$ such that

$$
-\int_{\Omega} u \Delta \zeta=\int_{\Omega} f \zeta d x-\int_{\partial \Omega} \frac{\partial \zeta}{\partial \mathbf{n}} \varphi d S
$$

for any $\zeta \in C_{c}^{1,1}(\bar{\Omega})$. Moreover there exists $C=C(\Omega)>0$ such that

$$
\|u\|_{L^{1}(\Omega)} \leq C\left(\left\|\rho_{\partial \Omega} f\right\|_{L^{1}(\Omega)}+\|\varphi\|_{L^{1}(\partial \Omega)}\right) .
$$

Finally u satisfies

$$
-\int_{\Omega}|u| \Delta \zeta+\int_{\partial \Omega} \frac{\partial \zeta}{\partial \mathbf{n}}|\varphi| d S \leq \int_{\Omega} f \zeta \operatorname{sgn}(u) d x,
$$

and

$$
-\int_{\Omega} u^{+} \Delta \zeta+\int_{\partial \Omega} \frac{\partial \zeta}{\partial \mathbf{n}} \varphi^{+} d S \leq \int_{\Omega} f \zeta \operatorname{sgn}^{+}(u) d x
$$

for any $\zeta \in C_{c}^{1,1}(\bar{\Omega}), \zeta \geq 0$.

Definition 1.2. Let $\mu \in \mathfrak{M}(\partial \Omega)$. A function $u \in L^{1}(\Omega)$ is a solution of

$$
\begin{aligned}
-\Delta u+g(x, u) & =0 \text { in } \Omega, \\
u & =\mu \text { on } \partial \Omega,
\end{aligned}
$$

if $g(., u) \in L^{1}\left(\Omega ; \rho_{\partial \Omega} d x\right)$ and

$$
\int_{\Omega}(-u \Delta \zeta+g(x, u) \zeta) d x=-\int_{\partial \Omega} \frac{\partial \zeta}{\partial \mathbf{n}} d \mu(y),
$$

for any $\zeta \in C_{c}^{1,1}(\bar{\Omega})$.

Uniqueness is a straightforward consequence of (1.5). In the case where $g(x, r)=g(r)$ and $\mu \in L^{1}(\partial \Omega)$, existence of a solution to (1.7) is due to Brezis [5]. If $g$ is continuous in $\bar{\Omega} \times \mathbb{R}$, the proof of Brezis result goes through without any difficulty. If $x \mapsto g(x, r)$ is merely continuous in $\Omega$ and unbounded near $\partial \Omega$, problem (1.7) may not have any solution, even with very regular data $\mu$.

Definition 1.2. A measure $\mu \in \mathfrak{M}(\partial \Omega)$ is g-admissible if

$$
g\left(., \mathbb{P}_{|\mu|}\right) \in L^{1}\left(\Omega ; \rho_{\partial \Omega} d x\right) .
$$

The two next results can be found in [27] 
Proposition 1.1. Let $g \in \mathcal{G}_{0}$ and $\mu \in \mathfrak{M}(\partial \Omega)$ be g-admissible. Then problem (1.7) possesses a unique solution $u_{\mu}$. Moreover the mapping $\mu \mapsto u_{\mu}$ is increasing and continuous from $\mathfrak{M}(\partial \Omega)$ endowed with the total variation norm into $C(\Omega)$ with the local uniform topology.

Proposition 1.2. Let $g \in \mathcal{G}_{0}$ satisfy

$$
g(., c) \in L^{1}\left(\Omega ; \rho_{\partial \Omega} d x\right), \quad \forall c \in \mathbb{R} .
$$

Then for any $\mu \in L^{1}(\partial \Omega)$, problem (0.4) admits a unique solution.

Let $d H_{N-1}$ be the (N-1)-dimensional Hausdorff measure. If $\mu \in \mathfrak{M}(\partial \Omega)$ we denote by $\mu_{R}$ (resp. $\mu_{s}$ ) its regular (resp. singular) part in the Lebesgue decomposition

$$
\mu=\mu_{R}+\mu_{s}
$$

where $\mu_{R} \prec d H_{N-1}$ ) and $\mu_{s} \perp \mu_{R}$. A variant of the next result can be found in [25].

Proposition 1.3. Assume $g \in \mathcal{G}_{0}$ satisfies (1.10) and

$$
|g(x, 2 r)| \leq K(|g(x, r)|+\ell(x), \forall(x, r) \in \Omega \times \mathbb{R}
$$

for some fixed $K>0$ and $\ell \in L^{1}\left(\Omega ; \rho_{\partial \Omega} d x\right)$. If $\mu \in \mathfrak{M}(\partial \Omega), \mu=\mu_{R}+\mu_{s}$ and $\mu_{s}$ is $g$-admissible, then the conclusions of Proposition 1.1 still hold.

ProOF. First notice that relation (1.11), called the $\Delta_{2}$-condition, implies

$$
\left|g\left(x, r+r^{\prime}\right)\right| \leq K\left(|g(x, r)|+\left|g\left(x, r^{\prime}\right)\right|\right)+\ell(x), \forall\left(x, r, r^{\prime}\right) \in \Omega \times \mathbb{R}^{2} .
$$

STEP 1. Assume that $\mu$ is nonnegative, and so are $\mu_{R}$ and $\mu_{s}$. Let $\left\{\mu_{R, n}\right\}$ be a sequence of smooth functions on $\partial \Omega$ converging to $\mu_{R}$ in $L^{1}(\partial \Omega)$. Since $\mathbb{P}_{\mu_{R, n}}$ is bounded, $g(x,$.$) is nondecreasing and (1.10) is satisfied, it follows from$ (1.12) that $\mu_{n}=\mu_{R, n}+\mu_{S}$ is $g$-admissible. Let $u_{n}$ and $v_{n}$ be the solutions of (0.4) with respective measure boundary data $\mu_{n}$ and $\mu_{R, n}$. Applying (1.5) with $u=v_{n}-v_{p}, f=-g\left(., v_{n}\right)+g\left(., v_{p}\right)$ and $\zeta=\mathbb{P}_{1}$, we obtain

$$
\left\|v_{n}-v_{p}\right\|_{L^{1}(\Omega)}+\left\|\rho_{\partial \Omega}\left(g\left(., v_{n}\right)-g\left(., v_{p}\right)\right)\right\|_{L^{1}(\Omega)} \leq C\left\|\mu_{R, n}-\mu_{R, p}\right\|_{L^{1}(\partial \Omega)} .
$$

Thus $\left\{v_{n}\right\}$ and $\left\{g\left(., v_{n}\right\}\right.$ converge to $v$ and $g(., v)$, respectively in $L^{1}(\Omega)$ and $L^{1}\left(\Omega ; \rho_{\partial \Omega} d x\right)$. Furthermore $v$ is the solution of $(0.4)$ with measure boundary data $\mu_{R}$. Because $v_{n}+\mathbb{P}_{\mu_{S}}$ is a supersolution of $(0.1)$ with boundary data $\mu_{n}$, there also holds

$$
0 \leq u_{n} \leq v_{n}+\mathbb{P}_{\mu_{S}},
$$


thus $\left\{u_{n}\right\}$ is uniformly integrable in $L^{1}(\Omega)$, and also locally compact in the $C_{\text {loc }}^{1}(\Omega)$ topology, by the elliptic equations regularity theory. Since inequality (1.12) leads to

$$
0 \leq g\left(x, u_{n}\right) \leq K\left(g\left(x, \mathbb{P}_{\mu_{S}}\right)+g\left(x, v_{n}\right)\right)+\ell(x)
$$

it follows from the assumption on $\mu_{S}$ that the sequence $\left\{g\left(., u_{n}\right)\right\}$ is uniformly integrable in $L^{1}\left(\Omega ; \rho_{\partial \Omega} d x\right)$. By the Vitali theorem $u_{n_{k}} \rightarrow u$ and $g\left(., u_{n_{k}}\right) \rightarrow$ $g(., u)$ respectively in $L^{1}(\Omega)$ and $L^{1}\left(\Omega ; \rho_{\partial \Omega} d x\right)$ and $u$ is the solution of $(0.4)$.

STEP 2. Let $\tilde{\mu}_{R, n}$ and $\bar{\mu}_{R, n}$ be smooth $L^{1}$-approximations of $\mu_{R}^{+}$and $\mu_{R}^{-}$, and denote by $u_{n}, \tilde{v}_{n}$ and $\bar{v}_{n}$ the solutions of (0.4) with respective measure boundary data $\mu_{n}=\tilde{\mu}_{R, n}+\bar{\mu}_{R, n}+\mu_{S}, \tilde{\mu}_{R, n}$ and $-\bar{\mu}_{R, n}$. By monotonicity and (1.12) there holds

$$
\bar{v}_{n}-\mathbb{P}_{\mu_{S}^{-}} \leq u_{n} \leq \tilde{v}_{n}+\mathbb{P}_{\mu_{S}^{+}}
$$

and

$K\left(g\left(x,-\mathbb{P}_{\mu_{S}^{-}}\right)+g\left(x, \bar{v}_{n}\right)\right)-\ell(x) \leq g\left(x, u_{n}\right) \leq K\left(g\left(x, \mathbb{P}_{\mu_{S}^{+}}\right)+g\left(x, \tilde{v}_{n}\right)\right)+\ell(x)$.

Since $\bar{v}_{n}, \tilde{v}_{n}, g\left(., \tilde{v}_{n}\right)$ and $g\left(., \bar{v}_{n}\right)$ inherit the uniform integrability properties of Step 1, we conclude again by the Vitali theorem.

Let $u_{\mu}$ denote the solution of (0.4) with boundary data $\mu$. The $g$ admissibility condition on $\mu$ does not imply the weak continuity of the mapping $\mu \mapsto u_{\mu}$, thus a more uniform assumption is needed.

Definition 1.3. We denote by $\mathcal{H G}_{0}$ the subset of the $g \in \mathcal{G}_{0}$ such that there exist two continuous, nondecreasing and nonnegative functions $h$ and $f$ defined on $\mathbb{R}_{+}$, with the property

$$
\begin{gathered}
0 \leq|g(x, r)| \leq h\left(\rho_{\partial \Omega}(x)\right) f(|r|), \quad \forall(x, r) \in \Omega \times \mathbb{R}, \\
\int_{0}^{1} h(s) f\left(\sigma s^{1-N}\right) s^{N} d s<\infty, \quad \forall \sigma \geq 0
\end{gathered}
$$

$$
\text { either } h(s)=s^{\alpha}, \text { for some } \alpha \geq 0 \text {, or } f \text { is convex }
$$

The main result of this section is an existence and stability theorem which extends a previous one due to Gmira and Véron [15]. The technique involved is based upon the use of Marcinkiewicz spaces first introduced by Benilan and Brezis [3], [6] for solving semilinear equations with right-hand side measure.

THEOREM 1.1. Let $g \in \mathcal{H} \mathcal{G}_{0}$. Then any measure $\mu$ on $\partial \Omega$ is g-admissible. Moreover, if $\left\{\mu_{n}\right\} \subset \mathfrak{M}(\partial \Omega)$ converges to $\mu$ in the weak sense of measures, the corresponding solutions $u_{\mu_{n}}$ of (0.4) with boundary data $\mu_{n}$ converge to $u_{\mu}$ locally uniformly in $\Omega$ and $g\left(., u_{\mu_{n}}\right) \rightarrow g\left(., u_{\mu}\right)$ in $L^{1}\left(\Omega ; \rho_{\partial \Omega} d x\right)$. 
Proof. Step 1. The Marcinkiewicz space framework. For any nonnegative locally bounded Borel measure $\beta$ in $\Omega$ and real number $p>1$, we denote

$$
\left.M^{p}(\Omega ; d \beta)=\left\{v \in L_{\mathrm{loc}}^{1}(\Omega ; d \beta):\|v\|_{M^{p}(\Omega ; d \beta)}\right\}<\infty\right\},
$$

where

$$
\|v\|_{M^{p}(\Omega ; d \beta)}=\inf \left\{c \in[0, \infty] \text { s.t. } \int_{K}|v| d \beta \leq c\left(\int_{K} d \beta\right)^{1-1 / p} \forall K \subset \Omega, K \text { Borel }\right\}
$$

Besides the classical imbedding of $M^{p}(\Omega ; d \beta)$ into $L_{\mathrm{loc}}^{\tilde{p}}(\Omega ; d \beta)$ for any $1 \leq$ $\tilde{p}<p$, the next inequality plays an important role

$$
\left.C(p)\|v\|_{M^{p}(\Omega ; d \beta)}\right\} \leq \sup _{\lambda>0}\left\{\lambda^{p} \int_{\{|u|>\lambda\}} d \beta\right\} \leq\|v\|_{M^{p}(\Omega ; d \beta)} .
$$

Moreover the following estimates are proved in [16]: there exists $K=K(\Omega)>0$ such that for any $v \in \mathfrak{M}(\partial \Omega)$,

$$
\begin{aligned}
\left\|\mathbb{P}_{\nu}\right\|_{M^{(N+1) /(N-1)}\left(\Omega ; \rho_{\partial \Omega} d x\right)} & \leq K\|v\|_{L^{1}(\partial \Omega)}, \\
\left\|\mathbb{P}_{\nu}\right\|_{M^{N /(N-1)}(\Omega)} & \leq K\|v\|_{L^{1}(\partial \Omega)}, \\
\left\|\mathbb{P}_{\nu}\right\|_{L^{\infty}\left(\Omega_{r}^{c}\right)} & \leq K r^{1-N}\|v\|_{L^{1}(\partial \Omega)},
\end{aligned}
$$

where $\Omega_{r}=\left\{x \in \Omega: \rho_{\partial \Omega}(x) \leq r\right\}$, and $\Omega_{r}^{c}=\Omega \backslash \bar{\Omega}_{r}=\left\{x \in \Omega: \rho_{\partial \Omega}(x)>r\right\}$.

STEP 2. We claim that there exist two positive constants $C_{1}=C_{1}(\Omega)$ and $C_{2}=C_{2}(N)$ such that for any $a \in \partial \Omega$ and $\lambda>0$

$$
\beta_{a}(\lambda)=\int_{\Gamma_{a}(\lambda)} h(\rho(x)) \rho(x) d x \leq C_{2} \int_{0}^{\left(C_{1} / \lambda\right)^{1 /(N-1)}} h(s) s^{N} d s,
$$

where

$$
\Gamma_{a}(\lambda)=\{x \in \Omega: P(x, a)>\lambda\} .
$$

Since

$$
C_{1}^{-1} \rho_{\partial \Omega}(x)|x-a|^{-N} \leq P(x, a) \leq C_{1} \rho_{\partial \Omega}(x)|x-a|^{-N},
$$

for some $C_{1}>0$ independent of $(x, a) \in \Omega \times \partial \Omega$,

$$
\Gamma_{a}(\lambda) \subset\left\{x \in \Omega: \rho_{\partial \Omega}(x)|x-a|^{-N}>\lambda / C_{1}\right\} \subset \Omega \cap B_{r_{\lambda}}(a)
$$


with $r_{\lambda}=\left(C_{1} / \lambda\right)^{1 /(N-1)}$. Since $h$ is nondecreasing,

$$
\int_{\Gamma_{a}(\lambda)} h(\rho(x)) \rho(x) d x \leq \int_{B_{r_{\lambda}}(a)} h(|x|) \rho(|x|) d x=\left|S^{N-1}\right| \int_{0}^{r_{\lambda}} h(s) s^{N} d s,
$$

which implies (1.25).

SteP 3. Let $G \subset \Omega$ be a Borel subset, then for any $m>0, \lambda>0$, and $a \in \partial \Omega$ there holds

$$
\begin{aligned}
\int_{G} h\left(\rho_{\partial \Omega}\right) f\left(m P(., a) \rho_{\partial \Omega} d x \leq f(\lambda) \int_{G} h\left(\rho_{\partial \Omega}\right) \rho_{\partial \Omega} d x\right. \\
\quad+C_{3} m^{(N+1) /(N-1)} \int_{\lambda}^{\infty} f(s) h\left(\left(m C_{1} / s\right)^{1 /(N-1))} s^{-2 N /(N-1)} d s\right.
\end{aligned}
$$

with $C_{3}=C_{3}(N)>0$. Actually,

$$
\begin{aligned}
\int_{G} h\left(\rho_{\partial \Omega}(x)\right) f\left(m P(x, a) \rho_{\partial \Omega}(x) d x=\right. & \int_{G \cap\{P(x, a) \leq \lambda / m\}} h\left(\rho_{\partial \Omega}(x)\right) f\left(m P(x, a) \rho_{\partial \Omega}(x) d x\right. \\
& +\int_{G \cap \Gamma_{a}(\lambda / m)} h\left(\rho_{\partial \Omega}(x)\right) f\left(m P(x, a) \rho_{\partial \Omega}(x) d x .\right.
\end{aligned}
$$

Since $f$ is nondecreasing,

$$
\int_{G \cap\{P(x, a) \leq \lambda / m\}} f\left(m P(x, a) h\left(\rho_{\partial \Omega}(x)\right) \rho_{\partial \Omega}(x) d x \leq f(\lambda) \int_{G} h\left(\rho_{\partial \Omega}(x)\right) \rho_{\partial \Omega}(x) d x .\right.
$$

Moreover

$$
\int_{G \cap \Gamma_{a}(\lambda / m)} h\left(\rho_{\partial \Omega}(x)\right) f\left(m P(x, a) \rho_{\partial \Omega}(x) d x \leq-\int_{\lambda / m}^{\infty} f(m s) d \beta_{a}(s) .\right.
$$

But

$$
-\int_{\lambda / m}^{\infty} f(m s) d \beta_{a}(s)=f(\lambda) \beta_{a}(\lambda)+\int_{\lambda / m}^{\infty} \beta_{a}(s) d f(m s) .
$$

Using (1.25) in Step 2 infers

$$
-\int_{\lambda / m}^{\infty} f(m s) d \beta_{a}(s) \leq f(\lambda) \beta_{a}(\lambda)+C_{2} \int_{\lambda / m}^{\infty} \int_{0}^{\left(C_{1} / s\right)^{1 /(N-1)}} h(\tau) \tau^{N} d \tau d f(m s) .
$$

Since

$$
\begin{aligned}
& \int_{\lambda / m}^{\infty} \int_{0}^{\left(C_{1} / s\right)^{1 /(N-1)}} h(\tau) \tau^{N} d \tau d f(m s)=-f(\lambda) \int_{0}^{\left(m C_{1} / \lambda\right)^{1 /(N-1)}} h(s) s^{N} d s \\
& \quad+\frac{C_{1}^{(N+1) /(N-1)}}{N-1} \int_{\lambda / m}^{\infty} h\left(\left(C_{1} / s\right)^{1 /(N-1)}\right) s^{-2 N /(N-1)} f(m s) d s,
\end{aligned}
$$


(1.27) follows by change of variable, with $C_{3}=C_{2} C_{1}^{(N+1) /(N-1)}$. It is important to notice that this integral is convergent because of (1.16).

STEP 4. Suppose $f$ is convex, then for any $\mu \in \mathfrak{M}^{+}(\partial \Omega)$ with total mass $m$ and any Borel subset $G \subset \Omega$, there holds

$$
\begin{aligned}
\int_{G} f\left(\mathbb{P}_{\mu}\right) h\left(\rho_{\partial \Omega}\right) \rho_{\partial \Omega} d x \leq f(\lambda) \int_{G} h\left(\rho_{\partial \Omega}\right) \rho_{\partial \Omega} d x \\
\quad+C_{3} m^{(N+1) /(N-1)} \int_{\lambda}^{\infty} f(s) h\left(\left(m C_{1} / s\right)^{1 /(N-1)}\right)^{-2 N /(N-1)} d s .
\end{aligned}
$$

First, let us assume that

$$
\mu=m \sum_{i=1}^{k} \theta_{i} \delta_{a_{i}}
$$

for some $a_{i} \in \partial \Omega$ and $\theta_{i}>0$ with $\sum_{i=1}^{k} \theta_{i}=1$. Then

$$
\mathbb{P}_{\mu}(x)=m \sum_{i=1}^{k} \theta_{i} P\left(x, a_{i}\right) .
$$

Since

$$
\begin{gathered}
f\left(\mathbb{P}_{\mu}(x)\right)=f\left(m \sum_{i=1}^{k} \theta_{i} P\left(x, a_{i}\right)\right) \leq \sum_{i=1}^{k} \theta_{i} f\left(m P\left(x, a_{i}\right)\right. \\
\int_{G} f\left(\mathbb{P}_{\mu}\right) h\left(\rho_{\partial \Omega}\right) \rho_{\partial \Omega} d x \leq \sum_{i=1}^{k} \theta_{i} \int_{G} f\left(m P\left(x, a_{i}\right) h\left(\rho_{\partial \Omega}\right) \rho_{\partial \Omega} d x .\right.
\end{gathered}
$$

Therefore (1.28) follows from (1.27).

For a general nonnegative measure $\mu$ with total mass $m$, there exists a sequence of finite combinations of positive Dirac measures $\mu_{n}$ with same total mass converging to $\mu$ in the weak sense of measures. Then $\mathbb{P}_{\mu_{n}}$ converges to $\mathbb{P}_{\mu}$ locally uniformly in $\Omega$ and in $L^{p}(\Omega)$ for any $1 \leq p<N /(N-1)$. Thus (1.28) follows by the Fatou's lemma.

STEP 5. Suppose $h(s)=s^{\alpha}$, then for any $\mu \in \mathfrak{M}^{+}(\partial \Omega)$ with total mass $m$ and any Borel subset $G \subset \Omega$, there holds

$$
\begin{aligned}
\int_{G} f & \left(\mathbb{P}_{\mu}\right) h\left(\rho_{\partial \Omega}\right) \rho_{\partial \Omega} d x \\
& \leq f(\lambda) \int_{G} \rho_{\partial \Omega}^{1+\alpha} d x+C_{7} m^{(N+1+\alpha) /(N-1)} \int_{\lambda}^{\infty} s^{-(2 N+\alpha) /(N-1)} f(s) d s .
\end{aligned}
$$

By Step 2 there exists $C_{4}=C_{4}(\Omega, \alpha)>0$ such that

$$
\beta_{a}(\lambda) \leq C_{4} \lambda^{(N+1+\alpha) /(N-1)}
$$


for any $\lambda>0$. Thus we derive an estimate of $P(., a)$ in $M^{(N+1+\alpha) /(N-1)}$,

$$
\int_{G} P(x, a) \rho_{\partial \Omega}^{1+\alpha} d x \leq C_{5}\left(\int_{G} \rho_{\partial \Omega}^{1+\alpha} d x\right)^{(2+\alpha) /(N+1+\alpha)} .
$$

Therefore

$$
\begin{aligned}
\left.\int_{G} \mathbb{P}_{\mu}(x)\right) \rho_{\partial \Omega}^{1+\alpha} d x & =\int_{\partial \Omega} d \mu(a) \int_{G} P(x, a) \rho_{\partial \Omega}^{1+\alpha} d x \\
& \leq\left(\int_{\partial \Omega} d \mu(a)\right) \max _{a \in \partial \Omega} \int_{G} P(x, a) \rho_{\partial \Omega}^{1+\alpha} d x
\end{aligned}
$$

From this estimate follows

$$
\left.\int_{G} \mathbb{P}_{\mu}(x)\right) \rho_{\partial \Omega}^{1+\alpha} d x \leq C_{5}\|\mu\|_{L^{1}(\partial \Omega)}\left(\int_{G} \rho_{\partial \Omega}^{1+\alpha} d x\right)^{(2+\alpha) /(N+1+\alpha)}
$$

Now

$$
\begin{aligned}
\int_{G} f\left(\mathbb{P}_{\mu}\right) h\left(\rho_{\partial \Omega}\right) \rho_{\partial \Omega} d x & =\int_{G} f\left(\mathbb{P}_{\mu}\right) \rho_{\partial \Omega}^{1+\alpha} d x \\
& \leq f(\lambda) \int_{G} \rho_{\partial \Omega}^{1+\alpha} d x+\int_{\left\{\mathbb{P}_{\mu}>\lambda\right\}} f\left(\mathbb{P}_{\mu}\right) \rho_{\partial \Omega}^{1+\alpha} d x
\end{aligned}
$$

But

$$
\int_{\left\{\mathbb{P}_{\mu}>\lambda\right\}} f\left(\mathbb{P}_{\mu}\right) \rho_{\partial \Omega}^{1+\alpha} d x=-\int_{\lambda}^{\infty} f(s) d \beta^{\mu}(s)
$$

where

$$
\beta^{\mu}(s)=\int_{\Gamma^{\mu}(s)} \rho_{\partial \Omega}^{1+\alpha} d x \quad \text { with } \quad \Gamma^{\mu}(s)=\left\{x \in \Omega: \mathbb{P}_{\mu}(x)>s\right\}
$$

Moreover

$$
\beta^{\mu}(s) \leq C_{6} m^{(N+1+\alpha) /(N-1)} \lambda^{-(N+1+\alpha) /(N-1)}
$$

by (1.30), with $G=\Gamma^{\mu}(\lambda)$ and $C_{6}=C_{5}^{(N+1+\alpha) /(N-1)}$. Therefore

$$
\begin{aligned}
-\int_{\lambda}^{\infty} f(s) d \beta^{\mu}(s)= & f(\lambda) \beta^{\mu}(\lambda)+\int_{\lambda}^{\infty} \beta^{\mu}(s) d f(s) \\
\leq & C_{6} m^{(N+1+\alpha) /(N-1)} \lambda^{-(N+1+\alpha) /(N-1)} f(\lambda) \\
& +C_{6} m^{(N+1+\alpha) /(N-1)} \int_{\lambda}^{\infty} s^{-(N+1+\alpha) /(N-1)} d f(s), \\
\leq & C_{7} m^{(N+1+\alpha) /(N-1)} \int_{\lambda}^{\infty} s^{-(2 N+\alpha) /(N-1)} f(s) d s
\end{aligned}
$$


where $C_{7}=(N+1+\alpha) C_{6} m^{(N+1+\alpha) /(N-1)}$. Combining (1.32) and (1.33) yields (1.29).

If we take $G=\Omega$ in (1.27) and (1.29) we derive that (1.9) holds with $\mu$ and we conclude by Proposition 1.1. However those two estimates are much more powerfull since they leads to uniform-integrability properties.

STEP 6. Put $v_{n}=\mathbb{P}_{\left|\mu_{n}\right|}$ and $v=\mathbb{P}_{|\mu|}$. Then

$$
0 \leq\left|u_{n}\right| \leq v_{n} \text { and } 0 \leq|u| \leq v
$$

The fact that $u_{n}$ is locally bounded in $\Omega$ independently of $n$ follows from (1.24). Since $g$ is continuous, $g\left(., u_{n}\right)$ remains also locally bounded in $\Omega$. By the elliptic equations regularity theory there exists a subsequence $\left\{u_{n_{k}}\right\}$ and a $C^{1}(\Omega)$-function $u$ such that $u_{n_{k}} \rightarrow u$ in the $C_{\text {loc }}^{1}(\Omega)$-topology. This clearly implies that $u$ solves $(0.1)$ in $\Omega$.

STEP 7. We claim that $u$ is a solution of (0.4) with $\mu$ as boundary data. By the definition of the Marcinkiewicz norm,

$$
\begin{aligned}
\int_{\Omega}\left|u_{n_{k}}-u\right| d x & =\int_{\Omega_{r}^{c}}\left|u_{n_{k}}-u\right| d x+\int_{\Omega_{r}}\left|u_{n_{k}}-u\right| d x \\
& \leq \int_{\Omega_{r}^{c}}\left|u_{n_{k}}-u\right| d x+2\left\|u_{n_{k}}-u\right\|_{M^{N /(N-1)}(\Omega)}\left(\text { meas. } \Omega_{r}\right)^{1 / N}
\end{aligned}
$$

But $\left\|u_{n_{k}}-u\right\|_{M^{N /(N-1)}(\Omega)}$ remains bounded independently of $n_{k}$ by (1.22). Thus $u_{n_{k}} \rightarrow u$ in $L^{1}(\Omega)$. In order to prove that $g\left(., u_{n_{k}}\right) \rightarrow g(., u)$ in $L^{1}\left(\Omega, \rho_{\partial \Omega} d x\right)$ put $m_{n}=\int_{\partial \Omega} d\left|\mu_{n}\right|$ and let $G \subset \Omega$ be a Borel set. Because of (1.16) and (1.34) there holds

$$
\left|g\left(., u_{n}\right)\right| \leq f\left(v_{n}\right) h\left(\rho_{\partial \Omega}\right) .
$$

If $f$ is convex (Step 4) it follows

$$
\begin{aligned}
\int_{G}\left|g\left(., u_{n}\right)\right| \rho_{\partial \Omega} d x \leq f(\lambda) \int_{G} h\left(\rho_{\partial \Omega}\right) \rho_{\partial \Omega} d x \\
\quad+C_{3} m_{n}^{(N+1) /(N-1)} \int_{\lambda}^{\infty} f(s) h\left(\left(m_{n} C_{1} / s\right)^{1 /(N-1))} s^{-2 N /(N-1)} d s .\right.
\end{aligned}
$$

If $h(s)=s^{\alpha}$ (Step 5), then

$$
\begin{aligned}
& \int_{G}\left|g\left(., u_{n}\right)\right| \rho_{\partial \Omega} d x \\
& \quad \leq f(\lambda) \int_{G} \rho_{\partial \Omega}^{1+\alpha} d x+C_{7} m_{n}^{(N+1+\alpha) /(N-1)} \int_{\lambda}^{\infty} s^{-(2 N+\alpha) /(N-1)} f(s) d s .
\end{aligned}
$$


Because $\left\{m_{n}\right\}$ is bounded, for any $\varepsilon>0$, we first choose $\lambda>0$ large enough so that, for any $n \in \mathbb{N}$,

$$
C_{3} m_{n}^{(N+1) /(N-1)} \int_{\lambda}^{\infty} f(s) h\left(\left(m_{n} C_{1} / s\right)^{1 /(N-1)} s^{-2 N /(N-1)} d s \leq \varepsilon / 2\right.
$$

in the case $f$ is convex, or

$$
C_{7} m_{n}^{(N+1+\alpha) /(N-1)} \int_{\lambda}^{\infty} s^{-(2 N+\alpha) /(N-1)} f(s) d s \leq \varepsilon / 2
$$

in the case $h(s)=s^{\alpha}$. Then we take meas. $G$ small enough so that

$$
f(\lambda) \int_{G} h\left(\rho_{\partial \Omega}\right) \rho_{\partial \Omega} d x, \leq \varepsilon / 2
$$

and we conclude that

$$
\int_{G}\left|g\left(., u_{n}\right)\right| \rho_{\partial \Omega} d x \leq \varepsilon
$$

independently of $n$. Therefore $\left\{g\left(., u_{n}\right\}\right.$ is uniformly integrable for the measure $\rho_{\partial \Omega} d x$. Since $g$ is continuous and $u_{n} \rightarrow u$ in $\Omega$, it follows that $g\left(., u_{n}\right) \rightarrow$ $g(., u)$ in $L^{1}\left(\Omega, \rho_{\partial \Omega} d x\right)$. Letting $n \rightarrow \infty$ in the integral formulation of $u_{n}$ for (0.4) implies that $u$ solves (0.4) with $\mu$ as boundary data.

The next stability result is a straightforward extension of the previous result

Proposition 1.4. Let $(x, r) \mapsto g_{n}(x, r)$ be a sequence of functions in $\in C(\Omega \times$ $\mathbb{R})$, nondecreasing with respect to $r$, vanishing at $r=0$ for any $x \in \Omega$, and satisfying (1.16)-(1.18) uniformly with respect to $n$. If there exists $g \in C(\Omega \times \mathbb{R})$ such that $g_{n}(x, r) \rightarrow g(x, r)$ pointwise in $\Omega \times \mathbb{R}$, then $g$ satisfies (1.16). Moreover, if $\mu_{n} \in \mathfrak{M}(\partial \Omega)$ converges to $\mu$ in the weak sense of measures, the sequence of solutions $u_{\mu_{n}, g_{n}}$ of

$$
\begin{aligned}
-\Delta u_{n}+g_{n}\left(x, u_{n}\right) & =0 \quad \text { in } \Omega, \\
u_{n} & =\mu_{n} \text { on } \partial \Omega,
\end{aligned}
$$

converges locally uniformly in $\Omega$ to the solution $u_{\mu}$ of (0.4).

Proof. By assumption

$$
0 \leq g_{n}(x, r) \leq h\left(\rho_{\partial \Omega}(x)\right) f(r), \forall n \in \mathbb{N}^{*}, \forall(x, r) \in \bar{\Omega} \times \mathbb{R},
$$

and (1.16) holds. Then $g$ satisfies the same upper bound. Since $0 \leq\left|u_{n}\right| \leq \mathbb{P}_{\left|\mu_{n}\right|}$, the inequalities (1.35)-(1.36) hold with $g$ replaced by $g_{n}$ which infers the uniform integrability of $\left\{g_{n}\left(., u_{n}\right)\right\}$ in $L^{1}\left(\Omega ; \rho_{\partial \Omega} d x\right)$. The rest of the proof is similar to the one of Proposition 1.1. 


\section{2. - The extended boundary trace}

If $\Omega$ is a $C^{3}$ bounded domain in $\mathbb{R}^{N}$ and $x \in \partial \Omega$, let $\mathbf{n}_{x}$ denote the normal pointing outward unit vector at $x$. Let us recall some notations and definitions from [25]. The mapping $\Pi$ from $\partial \Omega \times(0, \infty)$ into $\mathbb{R}^{N}$ is defined by

$$
\Pi(x, t)=x-t \mathbf{n}_{x} \quad \forall(x, t) \in \partial \Omega \times(0,1) .
$$

It is known that there exists $0<\beta_{0}$ such that $\Pi$ is a diffeomorphism from $\partial \Omega \times\left[0, \beta_{0}\right)$ onto

$$
\Omega_{\beta_{0}}=\left\{x \in \Omega: \rho_{\partial \Omega}(x)<\beta_{0}\right\} .
$$

In particular, for any $t \in\left[0, \beta_{0}\right)$ the set

$$
\Sigma_{t}=\left\{y=x-t \mathbf{n}_{x}: x \in \partial \Omega\right\}
$$

is diffeomorphic to $\partial \Omega=\Sigma_{0}$ (for the sake of simplicity, we shall denote $\Sigma_{0}=\Sigma$ ), and for any $y \in \Sigma_{t}, \rho_{\partial \Omega}(y)=t$. If $U \subset \partial \Omega$, we denote

$$
U_{t}=\left\{y=x-t \mathbf{n}_{x}: x \in U\right\},
$$

and if $\zeta$ is a function defined in $U_{t}$,

$$
\zeta_{t}(y)=\zeta(x) \text { for any } y=x-t \mathbf{n}_{x} \in U_{t} .
$$

We denote by $\mathfrak{H}_{t}$ the mapping from $\Sigma_{t}$ to $\Sigma$ defined by $\mathfrak{H}_{t}(x)=\sigma(x)$ for $x \in \Sigma_{t}$. Thus $\mathfrak{H}_{t}^{-1}(x)=\Pi^{-1}(., t)$.

Given $t \in\left[0, \beta_{0}\right)$, a Borel measure $\mu$ and a function $f$ on $\Sigma_{t}$, we define a corresponding measure $\mu^{t}$ and function $f^{t}$ on $\Sigma$ by

$$
\begin{cases}\mu^{t}(E)=\mu\left(\mathfrak{H}_{t}^{-1}(E)\right), & \forall E \subset \Sigma, E \text { Borel, } \\ f^{t}(\sigma)=f\left(\sigma-t \mathbf{n}_{\sigma}\right), & \forall \sigma \in \Sigma .\end{cases}
$$

Then

$$
\mu \in \mathfrak{M}\left(\Sigma_{t}\right), f \in L^{1}\left(\Sigma_{t},|\mu|\right) \Longrightarrow\left\{\begin{array}{l}
f^{t} \in L^{1}\left(\Sigma,\left|\mu^{t}\right|\right), \\
\int_{\Sigma_{t}} f d \mu=\int_{\Sigma} f^{t} d \mu^{t}
\end{array}\right.
$$

This section is devoted to the definition and properties of the notion of extended boundary trace for nonnegative solutions of

$$
-\Delta u+g(x, u) \geq 0 \quad \text { in } \Omega,
$$

and of the associated equation

$$
-\Delta u+g(x, u)=0 \quad \text { in } \Omega .
$$


Definition 2.1. I- We say that a function $g \in \mathcal{G}_{0}$ is positively subcritical if for any $\mu \in \mathfrak{M}_{+}(\partial \Omega)$, problem (0.4) admits a solution $u_{\mu}$.

II- The function $g$ is said positively subcritical and stable if $\mu_{n} \rightarrow \mu$ weakly in $\mathfrak{M}_{+}(\partial \Omega)$ implies

$u_{\mu_{n}} \rightarrow u_{\mu}$ locally uniformly in $\Omega$ and $g\left(., u_{\mu_{n}}\right) \rightarrow g\left(., u_{\mu}\right)$ in $L^{1}\left(\Omega ; \rho_{\partial \Omega} d x\right)$.

REMARK 2.1. I- The convergence of $\mu_{n}$ to $\mu$ in the weak sense of measures implies that $\mathbb{P}_{\mu_{n}}$ converges to $\mathbb{P}_{\mu}$ in $L^{s}(\Omega)$ for any $1 \leq s<N /(N-1)$, by classical potential analysis. Because $0 \leq u_{\mu_{n}} \leq \mathbb{P}_{\mu_{n}}, u_{\mu_{n}} \rightarrow u_{\mu}$ in $L^{s}(\Omega)$ (by Vitali's theorem) and in the local uniform topology of $C(\Omega)$ ). If $\zeta \in C_{c}^{2}(\bar{\Omega})$, $\zeta \geq 0$, then

$$
\int_{\Omega}\left(-u_{\mu_{n}} \Delta \zeta+g\left(x, u_{\mu_{n}}\right) \zeta\right) d x=-\int_{\partial \Omega} \frac{\partial \zeta}{\partial \mathbf{n}} d \mu_{n}
$$

Since $u_{\mu_{n_{k}}} \rightarrow \tilde{u}$ and $g\left(., u_{\mu_{n_{k}}}\right) \rightarrow g(., \tilde{u})$ locally uniformly in $\Omega$, Fatou's lemma infers

$$
\int_{\Omega}(-\tilde{u} \Delta \zeta+g(x, \tilde{u}) \zeta) d x \leq-\int_{\partial \Omega} \frac{\partial \zeta}{\partial \mathbf{n}} d \mu,
$$

and there always holds $\tilde{u} \leq u_{\mu}$. If it is assumed that $\tilde{u}=u_{\mu}$, then

$$
\lim _{n \rightarrow \infty} \int_{\Omega} g\left(x, u_{\mu_{n}}\right) \zeta d x=\int_{\Omega} g\left(x, u_{\mu}\right) \zeta d x
$$

and this convergence holds for any $\zeta \in C_{c}(\bar{\Omega})$ such that $\zeta / \rho_{\partial \Omega}$ is bounded. However it does not imply weak convergence in $L^{1}\left(\Omega ; \rho_{\partial \Omega} d x\right)$. Notice that in such a case, weak convergence in $L^{1}\left(\Omega ; \rho_{\partial \Omega} d x\right)$ implies strong convergence by Dunford-Pettis and Vitali's theorems.

II- It follows by Theorem 1.1 that any $g \in \mathcal{H} \mathcal{G}_{0}$ is positively subcritical and stable.

Definition 2.2. Let $U$ be a relatively open subset of $\Sigma$ and $\mu \in \mathfrak{M}(U)$. We say that a function $v \in C(\Omega)$ admits $\mu$ for trace on $\mathcal{O}$, and we denote it by $\operatorname{Tr}_{U}(v)$, if

$$
\lim _{t \rightarrow 0} \int_{U_{t}} v(x) \phi(\sigma(x)) d S=\int_{U} \phi d \mu, \quad \forall \phi \in C_{c}(U) .
$$

It is proved in [27, Corollary 1.3] that the solution $u_{\mu}$ of (0.4) admits $\mu$ for trace on $\partial \Omega$. The role of nonnegative super-solutions is enlighted by the next result. 
Proposition 2.1. Let $g \in \mathcal{G}_{0}$ and $u \in C(\Omega)$ be a nonnegative solution of (2.6) in $\Omega$ such that $g(., u) \in L^{1}\left(\Omega, \rho_{\partial \Omega} d x\right)$. Then $u$ admits a boundary trace $\mu \in \mathfrak{M}_{+}(\partial \Omega)$. Moreover, if

$$
u^{*}=\sup \{v \in C(\Omega): 0 \leq v \leq u, v \text { solution of }(2.7)\}
$$

then $u^{*}$ is a solution of (2.7) and

$$
\operatorname{Tr}_{\partial \Omega}(u)=\operatorname{Tr}_{\partial \Omega}\left(u^{*}\right) .
$$

Proof. Let $\psi=\mathbb{G}_{g(., u)}$ be the Green potential of the function $g(., u)$. Then $\psi+u$ is nonnegative and super-harmonic. Therefore $\psi+u$ admits a boundary trace belonging to $\mathfrak{M}_{+}(\partial \Omega)$. Thus the same holds for $u$ since $\psi$ vanishes on $\partial \Omega$ and the boundary trace of $u$ is a nonnegative Radon measure. The construction of $u^{*}$ is performed in considering the sequence of smooth domains $\Omega_{n}(n \geq 1)$, defined by

$$
\Omega_{n}=\left\{x \in \Omega: \rho_{\partial \Omega}(x)>\beta_{n}\right\},
$$

where $0<\beta_{n+1}<\beta_{n}<\beta_{0}$ and $\beta_{n} \rightarrow 0$ as $n \rightarrow \infty$. For each $n$ we denote by $u_{n}$ the solution of

$$
\begin{aligned}
-\Delta u_{n}+g\left(x, u_{n}\right) & =0 \text { in } \Omega_{n}, \\
u_{n} & =u \text { on } \partial \Omega_{n} .
\end{aligned}
$$

Since $u$ is a super solution, $u \geq u_{n} \geq 0$ and consequently the sequence $\left\{u_{n}\right\}$ is decreasing. Therefore $u^{*}$ exists as the decreasing limit of the $u_{n}$. By the regularity theory of elliptic equations, the convergence holds in $C_{\mathrm{loc}}^{1}(\Omega)$ and $u^{*}$ satisfies (2.7). If $\tilde{u}$ is any nonnegative solution of (2.7) dominated by $u$, then $\tilde{u} \leq u^{*}=u_{n}$ on $\partial \Omega_{n}$, thus $\tilde{u} \leq u_{n}$ in $\Omega_{n}$. Letting $n \rightarrow \infty$ yields $\tilde{u} \leq u^{*}$. Clearly the correspondence $u \mapsto u^{*}$ which associates to a solution $u$ of (2.6) the largest solution of (2.7) dominated by $u$ inherits the following properties:

$$
u_{1} \leq u_{2} \Longrightarrow u_{1}^{*} \leq u_{2}^{*},
$$

and

$$
\left(u^{*}\right)^{*}=u^{*} .
$$

In the above construction, the boundary trace of $u$ plays no role. In order to prove that $\operatorname{Tr}_{\partial \Omega}\left(u^{*}\right)=\mu$, let $\zeta \in C_{c}^{1,1}(\bar{\Omega})$ and $\zeta_{n}$ be the solution of

$$
\begin{array}{cl}
-\Delta \zeta_{n}=-\Delta \zeta & \text { in } \Omega_{n}, \\
\zeta_{n}=0 & \text { on } \partial \Omega_{n} .
\end{array}
$$

Although $\zeta_{n} \notin C_{c}^{1,1}\left(\bar{\Omega}_{n}\right), \zeta_{n}$ remains uniformly bounded in $C_{c}^{1, \alpha}\left(\bar{\Omega}_{n}\right)$ for any $\alpha \in(0,1), \Delta \zeta_{n}$ is bounded and

$$
\int_{\Omega_{n}}\left(-u_{n} \Delta \zeta_{n}+g\left(x, u_{n}\right) \zeta_{n}\right) d x=-\int_{\Sigma_{\beta_{n}}} \frac{\partial \zeta_{n}}{\partial \mathbf{n}} u(y) d S,
$$


by approximation. We extend $u_{n}$ by putting the zero value outside $\bar{\Omega}_{n}$ and call $\tilde{u}_{n}$ this extension. For $\zeta_{n}$ we perform an extension by reflexion following the normal direction and define $\tilde{\zeta}_{n}$ thanks to the following formula

$$
\begin{aligned}
& \forall x \in \bar{\Omega} \backslash \Omega_{n} \text { with } \rho_{\partial \Omega}(x)=t_{x} \text { and } x=\sigma(x)-t_{x} \mathbf{n}_{\sigma(\mathbf{x})}, \\
& \tilde{\zeta}_{n}(x)=-\zeta_{n}\left(\sigma(x)-\left(2 \beta_{n}-t_{x}\right) \mathbf{n}_{\sigma(\mathbf{x})}\right) .
\end{aligned}
$$

Notice that we have to assume $2 \beta_{n} \leq \beta_{0}$. It follows by the elliptic equations regularity theory that there exist a subsequence $\zeta_{n_{k}}$ and some $\tilde{\zeta} \in C_{c}^{1, \alpha}(\bar{\Omega})$ such that $\zeta_{n_{k}} \rightarrow \tilde{\zeta}$ in $C^{1}(\bar{\Omega})$. By the uniqueness of the solution of the Dirichlet problem $\tilde{\zeta}=\zeta$, and the wole sequence $\left\{\zeta_{n}\right\}$ is convergent. Moreover

$$
0 \leq \tilde{u}_{n}(x) \leq u(x) \Longrightarrow 0 \leq g\left(x, \tilde{u}_{n}\right) \leq g(x, u), \quad \forall x \in \Omega .
$$

Thus the sequence $\left\{g\left(., \tilde{u}_{n}\right) \rho_{\partial \Omega}\right\}$ is uniformly integrable in $\Omega$. By Vitali's theorem

$$
\int_{\Omega}\left(-u^{*} \Delta \zeta+g\left(x, u^{*}\right) \zeta\right) d x=-\lim _{n_{k} \rightarrow 0} \int_{\Sigma_{\beta_{n_{k}}}} \frac{\partial \zeta_{n_{k}}}{\partial \mathbf{n}} u(y) d S=\int_{\partial \Omega} \frac{\partial \zeta}{\partial \mathbf{n}} d \mu .
$$

This indicates that $u^{*}$ is a solution of (0.4) with boundary $\mu$ and therefore $\operatorname{Tr}_{\partial \Omega}\left(u^{*}\right)=\mu$.

The key observation on which is based the definition of the boundary trace is the following

Proposition 2.2. Let $g \in \mathcal{G}_{0}$ is positively subcritical and $u$ a nonnegative solution of (2.6) in $\Omega$. If $\mu \in \mathfrak{M}_{+}(\partial \Omega)$ set $w_{\mu}=\min \left(u, u_{\mu}\right)$. Then $w_{\mu}$ satifies

$$
-\Delta w_{\mu}+g\left(., w_{\mu}\right) \geq 0 \quad \text { in } \Omega,
$$

and there exists $\gamma_{u}(\mu) \in \mathfrak{M}_{+}(\partial \Omega)$ such that $\operatorname{Tr}_{\partial \Omega}\left(w_{\mu}\right)=\gamma_{u}(\mu)$. The mapping $\mu \mapsto \gamma_{u}(\mu)$ is nondecreasing and $0 \leq \gamma_{u}(\mu) \leq \mu$. Moreover, if for any $x \in \Omega$ the function $r \mapsto g(x, r)$ is convex on $\mathbb{R}_{+}$, the mapping $\mu \mapsto \gamma_{u}(\mu)$ is concave on $\mathfrak{M}_{+}(\partial \Omega)$.

Proof. Let $\delta>0$ and $p$ be the $C^{1,1}$ even convex function defined on $\mathbb{R}$ by

$$
p(t)= \begin{cases}|t|-\delta / 2 & \text { for }|t| \geq \delta \\ t^{2} / 2 \delta & \text { for }|t| \leq \delta .\end{cases}
$$

Then $\omega_{\delta}=\frac{1}{2}\left(u+u_{\mu}-p\left(u-u_{\mu}\right)\right)$ satisfies

$$
\begin{aligned}
\Delta \omega_{\delta} & =\frac{1}{2}\left(\Delta u+\Delta u_{\mu}-p^{\prime}\left(u+u_{\mu}\right) \Delta\left(u-u_{\mu}\right)-p^{\prime \prime}\left(u-u_{\mu}\right)\left|D\left(u-u_{\mu}\right)\right|^{2}\right), \\
& \leq \frac{1}{2}\left(\Delta u+\Delta u_{\mu}-p^{\prime}\left(u+u_{\mu}\right) \Delta\left(u-u_{\mu}\right)\right)=F
\end{aligned}
$$


Put

$$
\begin{aligned}
& G_{1}=\left\{x \in \Omega:\left(u-u_{\mu}\right)(x)>\delta\right\}, \\
& G_{2}=\left\{x \in \Omega:\left(u-u_{\mu}\right)(x)<-\delta\right\}, \\
& G_{3}=\left\{x \in \Omega:\left|u-u_{\mu}\right|(x) \leq \delta\right\} .
\end{aligned}
$$

On $G_{1}, p^{\prime}\left(u-u_{\mu}\right)=1$ and

$$
F=\Delta u_{\mu}=g\left(., u_{\mu}\right)=g\left(., \omega_{\delta}-\delta / 4\right)
$$

On $G_{2}, p^{\prime}\left(u-u_{\mu}\right)=-1$ and

$$
F=\Delta u \leq g\left(., u_{\mu}\right)=g\left(., \omega_{\delta}-\delta / 4\right) \text {. }
$$

On $G_{3}, p^{\prime}\left(u-u_{\mu}\right)=\delta^{-1}\left(u-u_{\mu}\right)$ and

$$
\begin{aligned}
F & =\frac{1}{2}\left(1-\frac{u-u_{\mu}}{\delta}\right) \Delta u+\frac{1}{2}\left(1+\frac{u-u_{\mu}}{\delta}\right) \Delta u_{\mu} \\
& \leq\left(1-\frac{u-u_{\mu}}{\delta}\right) g(., u)+\frac{1}{2}\left(1+\frac{u-u_{\mu}}{\delta}\right) g\left(., u_{\mu}\right)
\end{aligned}
$$

By continuity of $r \mapsto g(x, r)$ there exists $\theta=\theta(x) \in[0,1]$ such that

$$
F \leq g\left(., \theta u+(1-\theta) u_{\mu)}\right) \leq g\left(., \omega_{\delta}+3 \delta / 4\right) \leq g(., v+\delta)
$$

Combining those inequalities infers

$$
\Delta \omega_{\delta} \leq g\left(., \omega_{\delta}+3 \delta / 4\right) \leq g\left(., u_{\mu}+\delta\right)
$$

If we let $\delta \rightarrow 0, \omega_{\delta} \rightarrow w_{\mu}=\min \left(u, u_{\mu}\right)$ and (2.12) holds in the sense of distributions in $\Omega$. Since $0 \leq g\left(., w_{\mu}\right) \leq g\left(., u_{\mu}\right), g\left(., w_{\mu}\right) \in L^{1}\left(\Omega ; \rho_{\partial \Omega}\right) d x$. For the last assertion let $\mu_{i} \in \mathfrak{M}_{+}(\partial \Omega)(i=1,2), \theta \in[0,1], \mu_{\theta}=\theta \mu_{1}+(1-\theta) \mu_{2}$ and $u_{\theta}=\theta u_{\mu_{1}}+(1-\theta) u_{\mu_{2}}$. Since

$$
g\left(x, u_{\theta}\right) \leq \theta g\left(x, u_{\mu_{1}}\right)+(1-\theta) g\left(x, u_{\mu_{2}}\right),
$$

there holds

$$
-\Delta u_{\theta}+g\left(x, u_{\theta}\right) \leq 0,
$$

and $u_{\theta} \leq u_{\mu_{\theta}}$ by the comparison principle between solutions of (0.4). Moreover,

$$
\begin{aligned}
u+u_{\theta}-\left|u-u_{\theta}\right| & =\theta\left(u+u_{\mu_{1}}\right)+(1-\theta)\left(u+u_{\mu_{2}}\right)-\left|\theta\left(u-u_{\mu_{1}}\right)+(1-\theta)\left(u-u_{\mu_{2}}\right)\right| \\
& \geq \theta\left(u+u_{\mu_{1}}-\left|u-u_{\mu_{1}}\right|\right)+(1-\theta)\left(u+u_{\mu_{2}}-\left|u-u_{\mu_{2}}\right|\right) \\
& =\theta \min \left\{u, u_{\mu_{1}}\right\}+(1-\theta) \min \left\{u, u_{\mu_{2}}\right\} .
\end{aligned}
$$


Thus

$$
\min \left\{u, u_{\mu_{\theta}}\right\} \geq \min \left\{u, u_{\theta}\right\} \geq \theta \min \left\{u, u_{\mu_{1}}\right\}+(1-\theta) \min \left\{u, u_{\mu_{2}}\right\}
$$

which implies

$$
\gamma_{u}\left(\theta \mu_{1}+(1-\theta) \mu_{2}\right) \geq \theta \gamma_{u}\left(\mu_{1}\right)+(1-\theta) \gamma_{u}\left(\mu_{2}\right)
$$

REMARK 2.2. It follows also from [8], [9] that $\Delta w_{\mu} \in L^{1}\left(\Omega ; \rho_{\partial \Omega} d x\right)$. Moreover

$$
\int_{\Omega}\left(-w_{\mu} \Delta \zeta+g\left(x, w_{\mu}\right)\right) d x=\int_{\Omega} \Phi \zeta d x-\int_{\partial \Omega} \frac{\partial \zeta}{\partial \mathbf{n}} d \gamma_{u}(\mu), \forall \zeta \in C_{c}^{1,1}(\bar{\Omega})
$$

where $\Phi=\left[-\Delta w_{\mu}+g\left(x, w_{\mu}\right)\right]$.

Proposition 2.3. Under the assumptions of Proposition 2.2 set

$$
v=\sup \left\{\gamma_{u}(\mu): \mu \in \mathfrak{M}_{+}(\partial \Omega)\right\}
$$

Then $v$ is a Borel measure on $\partial \Omega$.

PROOF. It is clear that $v$ is an outer measure in the sense that

$$
v(\emptyset)=0, \quad \text { and } v(A) \leq \sum_{k=1}^{\infty} v\left(A_{k}\right), \text { whenever } A \subset \bigcup_{k=1}^{\infty} A_{k}
$$

Let $A$ and $B \subset \partial \Omega$ be disjoint Borel subsets. In order to prove that

$$
v(A \cup B)=v(A)+v(B),
$$

we first notice that the relation holds if $\max \{v(A), v(B)\}=\infty$. Therefore we assume that $v(A)$ and $v(B)$ are finite. For $\varepsilon>0$ there exist two bounded positive measures $\mu_{1}$ and $\mu_{2}$ such that

$$
\gamma_{u}\left(\mu_{1}\right)(A) \leq v(A) \leq \gamma_{u}\left(\mu_{1}\right)(A)+\varepsilon / 2
$$

and

$$
\gamma_{u}\left(\mu_{1}\right)(B) \leq v(B) \leq \gamma_{u}\left(\mu_{1}\right)(B)+\varepsilon / 2
$$

Hence

$$
\begin{aligned}
v(A)+v(B) & \leq \gamma_{u}\left(\mu_{1}\right)(A)+\gamma_{u}\left(\mu_{2}\right)(B)+\varepsilon \\
& \leq \gamma_{u}\left(\mu_{1}+\mu_{2}\right)(A)+\gamma_{u}\left(\mu_{1}+\mu_{2}\right)(B)+\varepsilon \\
& =\gamma_{u}\left(\mu_{1}+\mu_{2}\right)(A \cup B)+\varepsilon \\
& \leq v(A \cup B)+\varepsilon .
\end{aligned}
$$


Therefore $v$ is a finitely additive measure. If $\left\{A_{k}\right\}(k \geq 0)$ is a sequence of of disjoint Borel sets and $A=\cup A_{k}$, then

$$
v(A) \geq v\left(\bigcup_{1 \leq k \leq n} A_{k}\right)=\sum_{k=1}^{n} v\left(A_{k}\right) \Longrightarrow v(A) \geq \sum_{k=1}^{\infty} v\left(A_{k}\right) .
$$

By (2.17), it implies that $v$ is a countably additive measure.

REMARK 2.3. The measure $v$ may not be regular. If $v(B)=\infty$ then $\nu(\mathcal{O})=\infty$ for any relatively open subset $\mathcal{O}$ containing $B$. On the other hand, if $v(B)<\infty$, there exists a sequence of positive Radon measures $\mu_{n}$ such that

$$
\gamma_{u}\left(\mu_{n}\right)(B) \uparrow \nu(B) \text { as } n \rightarrow \infty .
$$

Even if for each $n \in \mathbb{N}_{*}$ and $\epsilon>0$ there exists a relatively open subset $\mathcal{O}_{n, \epsilon}$ containing $B$ such that

$$
\gamma_{u}\left(\mu_{n}\right)\left(\mathcal{O}_{n, \epsilon}\right) \leq \gamma_{u}\left(\mu_{n}\right)(B)+\epsilon,
$$

there is no reason that there exists some open subset containing $B$ such that $\gamma_{u}\left(\mu_{n}\right)(\mathcal{O})$ would remain bounded independently of $n$.

Definition 2.3. The outer Borel measure $v$ defined by the above process is called the extended boundary trace of $u$ and denoted by $\operatorname{Tr}_{\partial \Omega}^{e}(u)$.

The next result shows that in the study of the extended boundary trace, it is always possible to replace the inequation by an equation.

Proposition 2.4. Let $g \in \mathcal{G}_{0}$ be positively subcritical. If $u$ is a nonnegative solution of (2.6) and $u^{*}$ is the largest solution of (0.1) dominated by $u$, then

$$
\operatorname{Tr}_{\partial \Omega}^{e}(u)=\operatorname{Tr}_{\partial \Omega}^{e}\left(u^{*}\right) .
$$

If, in addition, there exist an open domain $\mathcal{O} \subset \mathbb{R}^{N}$ and a nonnegative Radon measure $\tilde{\mu}$ on $\mathcal{O} \cap \partial \Omega$ such that

$$
\lim _{\beta \rightarrow 0} \int_{\Sigma_{\beta} \cap \mathcal{O}} u(x) \phi(\sigma(x)) d S=\int_{\mathcal{O} \cap \partial \Omega} \phi d \tilde{\mu} .
$$

for any $\phi \in C(\mathcal{O} \cap \partial \Omega)$, then

$$
\left.\operatorname{Tr}_{\partial \Omega}^{e}(u)\right|_{\partial \Omega \cap \mathcal{O}}=\tilde{\mu} .
$$

Proof. Because of the definition of the extended boundary trace, it is sufficient to prove that for any $\mu \in \mathfrak{M}_{+}(\partial \Omega)$,

$$
\gamma_{u}(\mu)=\gamma_{u^{*}}(\mu) .
$$


Because $u^{*} \leq u$, then $\min \left\{u^{*}, u_{\mu}\right\} \leq \min \left\{u, u_{\mu}\right\}$ and $\gamma_{u^{*}}(\mu) \leq \gamma_{u}(\mu)$. Conversely

$$
u \geq \min \left\{u, u_{\mu}\right\} \Longrightarrow u^{*} \geq\left[\min \left\{u, u_{\mu}\right\}\right]^{*}
$$

and

$$
u_{\mu} \geq \min \left\{u, u_{\mu}\right\} \Longrightarrow u_{\mu}^{*}=u_{\mu} \geq\left[\min \left\{u, u_{\mu}\right\}\right]^{*}
$$

by (2.10). Therefore

$$
\min \left\{u^{*}, u_{\mu}\right\} \geq\left[\min \left\{u, u_{\mu}\right\}\right]^{*},
$$

and

$$
\left[\min \left\{u^{*}, u_{\mu}\right\}\right]^{*} \geq\left(\left[\min \left\{u, u_{\mu}\right\}\right]^{*}\right)^{*}=\left[\min \left\{u, u_{\mu}\right\}\right]^{*},
$$

by (2.11). By Proposition 2.1, $\min \left\{u, u_{\mu}\right\}$ and $\left[\min \left\{u, u_{\mu}\right\}\right]^{*}$ have the same boundary trace, and the same holds $\min \left\{u^{*}, u_{\mu}\right\}$ and $\left[\min \left\{u^{*}, u_{\mu}\right\}\right]^{*}$. Therefore

$$
\gamma_{u} *(\mu) \geq \gamma_{u}(\mu)
$$

which implies (2.22).

For the second assertion, we assume that $u$ admits $\tilde{\mu}$ for boundary trace on $\mathcal{O} \cap \partial \Omega$. Let $\lambda \in \mathfrak{M}_{+}(\partial \Omega)$ and $\phi \in C_{c}(\partial \Omega \cap \mathcal{O}), \phi \geq 0$. Since

$$
\int_{\Sigma_{\beta} \cap \mathcal{O}} u(x) \phi(\sigma(x)) d S \geq \int_{\Sigma_{\beta} \cap \mathcal{O}} \min \left\{u(x), u_{\lambda}(x)\right\} \phi(\sigma(x)) d S,
$$

there holds, as $\beta \rightarrow 0$,

$$
\int_{\partial \Omega \cap \mathcal{O}} \phi d \tilde{\mu} \geq \int_{\partial \Omega \cap \mathcal{O}} \phi d \gamma_{u}(\lambda)
$$

thus

$$
\tilde{\mu} \geq\left.\operatorname{Tr}_{\partial \Omega}^{e}(u)\right|_{\partial \Omega \cap \mathcal{O}}
$$

Conversely, by reducing the set $\mathcal{O}$, we first suppose that $\tilde{\mu}$ is bounded and we extend it by 0 outside $\mathcal{O} \cap \partial \Omega$. We can also suppose that $\mathcal{O} \cap \Omega$ is $C^{2}$ and that $u \in L^{1}(\Omega \cap \partial \mathcal{O})$ by (2.20) and Fubini's theorem. Let $v=v_{\tilde{\mu}}^{\mathcal{O}}$ be the solution of

$$
\begin{aligned}
-\Delta v+g(x, v)=0 & \text { in } \mathcal{O} \cap \Omega, \\
v=0 & \text { in } \Omega \cap \partial \mathcal{O}, \\
v=\tilde{\mu} & \text { in } \partial \Omega \cap \mathcal{O} .
\end{aligned}
$$

Since $\left.u\right|_{\mathcal{O} \cap \Omega}$ satisfies the same equation, with the exception of the data on $\Omega \cap \partial \mathcal{O}$ which is an integrable nonnegative function, $\left.g(., u)\right|_{\mathcal{O} \cap \Omega} \in L^{1}\left(\mathcal{O} \cap \Omega ; \rho_{\partial(\mathcal{O} \cap \Omega)} d x\right)$ and

$$
v_{\tilde{\mu}}^{\mathcal{O}} \leq u \text { in } \mathcal{O} \cap \Omega
$$


by the maximum principle and [5]. If $\tilde{v}=\tilde{v}_{\tilde{\mu}}^{\mathcal{O}}$, is the extension of $v$ by zero in $\Omega \backslash(\mathcal{O} \cap \Omega)$, then

$$
\tilde{v} \leq u_{\tilde{\mu}} \leq \min \left\{u, u_{\tilde{\mu}}\right\}
$$

Therefore

$$
\int_{\mathcal{O}} \phi d \tilde{\mu} \leq \int_{\mathcal{O}} \phi d \gamma_{u}(\tilde{\mu})
$$

for any $\phi \in C_{c}(\partial \Omega \cap \mathcal{O})$. Clearly the same relation holds even if we no longer assume that $\tilde{\mu}$ is bounded. Thus

$$
\tilde{\mu}(E) \leq \gamma_{u}(\tilde{\mu})(E), \quad \forall E \subset \partial \Omega \cap \mathcal{O}, E \text { Borel },
$$

and consequently

$$
\tilde{\mu} \leq\left.\operatorname{Tr}_{\partial \Omega}^{e}(u)\right|_{\partial \Omega \cap \mathcal{O}}
$$

REMARK 2.4. The relation (2.20) means that $u$ admits a boundary trace in the usual sense on $\mathcal{O} \cap \partial \Omega$ which is precisely $\tilde{\mu}$. The reverse implication "(2.21) $\Longrightarrow(2.20)$ " holds under an additional strong stability assumption which will be developped in Section 4. However we can give a weaker form of this implication if $u^{*}$ is dominated by the minimal large solution of (2.7), whenever it exists.

Let us denote by $\lambda_{n}(n \geq 0)$ the measure $n \chi_{\partial \Omega} d S$ and

$$
u_{m}=\sup \left\{u_{\lambda_{n}}: n \in \mathbb{N}\right\} .
$$

If $u_{m}$ is locally bounded in $\Omega$, it is a solution of (2.7) which blows up on the boundary. In such a case it is called the minimal large solution. Depending upon the nonlinearity, $u_{m}$ may also be infinite in whole $\Omega$ or in part of $\Omega$. Moreover, by the maximum principle, it dominates any solution $u$ of the same equation which is obtained by approximation by solutions with finite values as boundary data.

Proposition 2.5. Let $g \in \mathcal{H}_{0}$ be positively subcritical. If $\operatorname{Tr}_{\partial \Omega}^{e}(u)$ is a bounded Borel measure and $u^{*} \leq u_{m}$, then

$$
\operatorname{Tr}_{\partial \Omega}^{e}\left(u^{*}\right)=\operatorname{Tr}_{\partial \Omega}\left(u^{*}\right) \in \mathfrak{M}_{+}(\partial \Omega) .
$$

Proof. By assumption $v=\operatorname{Tr}_{\partial \Omega}^{e}(u)$ is bounded. Thus there exists a sequence $\left\{\mu_{n}\right\} \subset \mathfrak{M}_{+}(\partial \Omega)$ such that

$$
\gamma_{u}\left(\mu_{n}\right)(1)=\gamma_{u^{*}}\left(\mu_{n}\right)(1)=\operatorname{Tr}_{\partial \Omega}\left(\left[\min \left\{u^{*}, u_{\mu_{n}}\right\}\right]^{*}\right)(1) \uparrow v(1),
$$

since $u$ and $u^{*}$ have the same extended boundary trace. Because the extended boundary trace is defined by a supremum over all measures, it can also be 
assumed that the regular part of $\mu_{n}$ is a.e. bounded from below by $n$. Let $\zeta \in C_{c}^{1,1}(\bar{\Omega})$ be the solution of

$$
\begin{aligned}
-\Delta \zeta & =1 \text { in } \Omega, \\
\zeta & =0 \text { on } \partial \Omega .
\end{aligned}
$$

By the definition of the boundary trace in $\mathfrak{M}_{+}(\partial \Omega)$,

$$
\begin{aligned}
\int_{\Omega}\left(\left[\min \left\{u^{*}, u_{\lambda_{n}}\right\}\right]^{*}+\zeta g\left(x,\left[\min \left\{u^{*}, u_{\lambda_{n}}\right\}\right]^{*}\right) d x\right. & =-\int_{\partial \Omega} \frac{\partial \zeta}{\partial \mathbf{n}} d \operatorname{Tr}_{\partial \Omega}\left(\left[\min \left\{u^{*}, u_{\lambda_{n}}\right\}\right]^{*}\right), \\
& \leq C \nu(1) .
\end{aligned}
$$

Since $u_{m}$ dominates $u^{*}, \lim _{n \rightarrow \infty} \min \left\{u^{*}, u_{\lambda_{n}}\right\}=u^{*}$ and

$$
u^{*} \in L^{1}(\Omega) \text {, and } g\left(., u^{*}\right) \in L^{1}\left(\Omega ; \rho_{\partial \Omega} d x\right) .
$$

It follows from [27, Corollary 1.3] that $u^{*}$ has a boundary trace, say $\mu^{*}$ in $\mathfrak{M}_{+}(\partial \Omega)$, and $u^{*}=u_{\mu^{*}}$. Consequently for any measure $\mu$ larger than $\mu^{*}$, $\min \left\{u_{\mu}, u_{\mu^{*}}\right\}=u_{\mu^{*}}$ and

$$
\operatorname{Tr}_{\partial \Omega}^{e}\left(u^{*}\right)=\operatorname{Tr}_{\partial \Omega}^{e}\left(u_{\mu^{*}}\right)=\sup _{\mu \geq \mu^{*}} \gamma_{u^{*}}(\mu)=\mu^{*}=\operatorname{Tr}_{\partial \Omega}\left(u^{*}\right) .
$$

REMARK 2.5. The previous result still holds if, in the domination assumption $u^{*} \leq u_{m}$, the function $u_{m}$ is no longer the minimal large solution, but any $\sigma$ moderate solution in the sense of Dynkin and Kuznetsov [12], that is a solution of (2.7) which is an increasing limit of solutions $u_{\mu_{n}}$ for some $\mu_{n} \in \mathfrak{M}_{+}(\partial \Omega)$

PROPOSITION 2.6. Let $g \in \mathcal{G}_{0}$ be positively subcritical and stable, and let $u$ be a nonnegative solution of (0.9). If $\left\{\mu_{n}\right\} \subset \mathfrak{M}_{+}(\partial \Omega)$ converges weakly to $\mu$, then $\limsup _{n \rightarrow \infty} \gamma_{u}\left(\mu_{n}\right) \leq \gamma_{u}(\mu)$. If we assume moreover that the sequence $\left\{\mu_{n}\right\}$ is nonincreasing, $\lim _{n \rightarrow \infty} \gamma_{u}\left(\mu_{n}\right)=\gamma_{u}(\mu)$.

PRoOF. Since $\mu_{n} \rightarrow \mu$ in the weak sense of measures on $\partial \Omega, u_{\mu_{n}} \rightarrow u_{\mu}$ locally uniformly in $\Omega$ by definition of the positive subcriticality and stability. Thus

$$
w_{\mu_{n}}=\min \left\{u^{*}, u_{\mu_{n}}\right\} \rightarrow w_{\mu}=\min \left\{u^{*}, u_{\mu}\right\} \text { in the } C_{\text {loc }}(\Omega) \text {-topology. }
$$

Since $u_{\mu_{n}} \leq \mathbb{P}_{\mu_{n}}$ and $P_{\mu_{n}} \rightarrow P_{\mu}$ in $L^{1}(\Omega)$, the convergence of $w_{\mu_{n}}$ to $w_{\mu}$ holds also in $L^{1}(\Omega)$. Moreover

$$
g\left(., w_{\mu_{n}}\right) \rightarrow g\left(., w_{\mu}\right)
$$

in $C_{\text {loc }}(\Omega)$. Since

$$
0 \leq g\left(., w_{\mu_{n}}\right) \leq g\left(., u_{\mu_{n}}\right)
$$


and

in $L^{1}\left(\Omega ; \rho_{\partial \Omega} d x\right)$,

$$
g\left(., u_{\mu_{n}}\right) \rightarrow g\left(., u_{\mu}\right)
$$

also in $L^{1}\left(\Omega ; \rho_{\partial \Omega} d x\right)$. Put $v_{n}^{*}=\left[w_{\mu_{n}}\right]^{*}$. By the elliptic equations regularity theory, $\left\{v_{n}^{*}\right\}$ remains bounded in $C_{\mathrm{loc}}^{1}(\Omega)$. Since $\gamma_{u}\left(\mu_{n}\right)$ is dominated by $\mu_{n}$ which is bounded let us consider a subsequence $\gamma_{u}\left(\mu_{n_{k}}\right)$ weakly convergent to some nonnegative measure $\lambda$. Up to an extraction of a subsequence, it is always possible to assume that $v_{n_{k}}^{*}$ converges (in the $C_{\text {loc }}(\Omega)$-topology) to $\bar{v}$. Clearly $\bar{v}$ is a solution of (0.1) in $\Omega$ and

$$
v_{n}^{*} \leq w_{\mu_{n}} \Longrightarrow \bar{v} \leq \lim _{n \rightarrow \infty} w_{\mu_{n}}=w_{\mu} .
$$

Therefore

$$
\bar{v}=\bar{v}^{*} \leq v^{*}=\left[w_{\mu}\right]^{*}
$$

Inasmuch

$$
v_{n_{k}}^{*} \rightarrow \bar{v} \text { in } L^{1}(\Omega) \text { and } g\left(., v_{n_{k}}^{*}\right) \rightarrow g(., \bar{v}) \text { in } L^{1}\left(\Omega ; \rho_{\partial \Omega} d x\right),
$$

(for the second relation we use $0 \leq g\left(., v_{n_{k}}^{*}\right) \leq g\left(., w_{\mu_{n}}\right)$, together with (2.29) and Vitali's theorem) and

$$
v_{n_{k}}+\mathbb{G}_{g\left(., v_{n_{k}}^{*}\right)}=\mathbb{P}_{\gamma_{u}\left(\mu_{n}\right)},
$$

it follows

$$
\bar{v}+\mathbb{G}_{g(., \bar{v})}=\mathbb{P}_{\lambda}
$$

But

As $\bar{v} \leq v^{*}$ and $g(., \bar{v}) \leq g\left(., v^{*}\right)$,

$$
\mathbb{P}_{\lambda} \leq \mathbb{P}_{\gamma_{u}(\mu)} \Longrightarrow \lambda \leq \gamma_{u}(\mu),
$$

which is the first assertion.

If we assume that $\left\{\mu_{n}\right\}$ is nonincreasing, the same holds with $\left\{u_{\mu_{n}}\right\},\left\{w_{\mu_{n}}\right\}$, $\left\{\gamma_{u}\left(\mu_{n}\right)\right\}$ and $\left\{v_{n}^{*}\right\}$. If $\mu_{n} \downarrow \mu$, any solution of (0.1) dominated by $w_{\mu}$ is dominated by $w_{\mu_{n}}$. Thus $v^{*} \leq v_{n}^{*}$ and $\bar{v}=\lim _{n \rightarrow \infty} v_{n}^{*}=v^{*}$ by (2.30) and (2.31).

A particularly important case deals with the choice $\mu=\lambda \delta_{a}$, with $a \in \partial \Omega$ and $\lambda>0$. Let $u=u_{\lambda \delta_{a}}$ be the solution of

$$
\begin{aligned}
-\Delta u+g(x, u)=0, & \text { in } \Omega, \\
u=\lambda \delta_{a}, & \text { on } \partial \Omega .
\end{aligned}
$$

Since $g(x,$.$) is nondecreasing, \lambda \mapsto u_{\lambda \delta_{a}}$ is increasing. Set

$$
u_{\infty, a}=\lim _{\lambda \rightarrow \infty} u_{\lambda \delta_{a}} .
$$

On any open subset of $\Omega$ where it is locally finite, $u_{\infty, a}$ is a solution of (0.1). 
Lemma 2.1. Let $a \in \partial \Omega, \lambda>0$ and $w_{\lambda \delta_{a}}=\min \left\{u, u_{\lambda \delta_{a}}\right\}$. Then

$$
\operatorname{Tr}_{\partial \Omega}\left(w_{\lambda \delta_{a}}\right)=\gamma_{u}\left(\lambda \delta_{a}\right)=\tilde{\gamma}_{u}(a, \lambda) \delta_{a}
$$

where $0 \leq \tilde{\gamma}_{u}(a, \lambda) \leq \lambda$. Moreover the mapping $\lambda \mapsto \tilde{\gamma}_{u}(a, \lambda)$ is nondecreasing, there exists

$$
\tilde{\gamma}_{u}(a)=\lim _{\lambda \rightarrow \infty} \tilde{\gamma}_{u}(a, \lambda)
$$

and

$$
\min \left(u, u_{\infty, a}\right) \geq u_{\tilde{\gamma}_{u}(a)} .
$$

Proof. Because $\operatorname{Tr}_{\partial \Omega}\left(w_{\lambda \delta_{a}}\right) \leq \lambda \delta_{a}$, this trace is concentrated at $a$ and achieves the form $\tilde{\gamma}_{u}(a, \lambda) \delta_{a}$. Moreover $\lambda \mapsto \tilde{\gamma}_{u}(a, \lambda)$ is nondecreasing as is the mapping $\lambda \mapsto \min \left(u, u_{\lambda \delta_{a}}\right)$. Since

$$
w_{\lambda \delta_{a}} \geq u_{\tilde{\gamma}_{u}(a, \lambda) \delta_{a}},
$$

and $u_{\lambda \delta_{a}}$ admits a limit, finite or not, when $\lambda \rightarrow \infty$, assertions (i) or (ii) follow.

The next result points out the role of $u_{\infty, a}$

Proposition 2.7. Let $g \in \mathcal{G}_{0}$ be positively subcritical. If $u$ is a nonnegative solution of (2.6) with boundary trace $v$ and $a \in \partial \Omega$, then

$$
u \geq u_{\infty, a} \Longrightarrow v(a)=\infty
$$

If we assume moreover that $g$ is positively subcritical and stable, then

$$
v(a)=\infty \Longrightarrow u \geq u_{\infty, a}
$$

Proof. Let $a \in \partial \Omega$ be such that

$$
v(a)=\infty
$$

Then for any relatively open subset $\mathcal{O} \subset \partial \Omega$ containing $a$,

$$
v(\mathcal{O})=\infty
$$

which means that there exists a sequence of positive Radon measures $\mu_{n}$ such that

$$
\lim _{n \rightarrow \infty} \gamma_{u}\left(\mu_{n}\right)(\mathcal{O})=\infty
$$

Without any restriction we can suppose that the sequence of restricted measures $\gamma_{u}^{\prime}\left(\mu_{n}\right)=\chi_{\mathcal{O}} \gamma_{u}\left(\mu_{n}\right)$ is increasing and

$$
u \geq u_{\gamma_{u}^{\prime}\left(\mu_{n}\right)},
$$


because $u \geq u_{\gamma_{u}\left(\mu_{n}\right)}$ and $\gamma_{u}\left(\mu_{n}\right) \geq \gamma_{u}^{\prime}\left(\mu_{n}\right)$. For any $k \in \mathbf{N}_{*}$, there exists $\epsilon_{n, k}>0$ such that, if we take $\mathcal{O}_{n, k}=B_{\epsilon_{n, k}}(a) \cap \partial \Omega$, there holds

$$
\gamma_{u}\left(\mu_{n}\right)\left(\mathcal{O}_{n, k}\right)=k
$$

Set $\mu_{n, k}=\chi_{\mathcal{O}_{n, k}} \gamma_{u}\left(\mu_{n}\right)$. Then

$$
\gamma_{u}\left(\mu_{n}\right) \geq \mu_{n, k} \Longrightarrow u_{\gamma_{u}\left(\mu_{n}\right)} \geq u_{\mu_{n, k}} .
$$

Since $\lim _{n \rightarrow \infty} \epsilon_{n, k}=0, \lim _{n \rightarrow \infty} \mu_{n, k}=k \delta_{a}$. Consequently

$$
u(x) \geq u_{k \delta_{a}}(x) .
$$

Letting $k \rightarrow \infty$ yields to the following implication

$$
v(a)=\infty \Longrightarrow u \geq u_{\infty a} .
$$

Conversely, assume $u \geq u_{\infty a}$, then for any $k>0, u \geq u_{k \delta_{a}}$. On one hand the boundary trace of $w_{k \delta_{a}}=\min \left\{u, u_{k \delta_{a}}\right\}$ is the measure $\gamma_{u}(a, k) \delta_{a}$. But $\min \left\{u, u_{k \delta_{a}}\right\}=u_{k \delta_{a}}$ implies $\gamma_{u}(a, k)=k$ and therefore $\gamma_{u}\left(k \delta_{a}\right)=k \delta_{a}$. By the definition of $v$,

$$
v(a) \geq \gamma_{u}\left(k \delta_{a}\right)(a)=k .
$$

Since this holds for any $k>0, v(a)=\infty$.

The characterisation of Borel subsets on which the boundary trace of $u$ takes finite values is less complete, however there holds

Proposition 2.8. Assume the assumptions of Proposition 2.2 are fulfilled and $u$ is a nonnegative solution of (2.6) with boundary trace $v$. If $\mathcal{O} \subset \partial \Omega$ is a relatively open subset of $\partial \Omega$ such that

$$
\int_{\mathcal{O}_{t}} u(y) d S_{t}
$$

remains bounded independently of $t \in\left(0, \beta_{0}\right]$, then $v(\mathcal{O})$ is finite.

PRoOF. Let $\mu$ be a nonnegative measure. Since $u_{\gamma_{u}(\mu)} \leq u$ in $\Omega$,

$$
\int_{\mathcal{O}_{t}} u_{\gamma_{u}(\mu)}(y) d S_{t} \leq \int_{\mathcal{O}_{t}} u(y) d S_{t} .
$$

Thus

$$
\gamma_{u}(\mu)(\mathcal{O}) \leq \sup _{0<\beta \leq \beta_{0}} \int_{\mathcal{O}_{t}} u(y) d S_{t}=M
$$

Therefore

$$
\nu(\mathcal{O}))=\sup _{\mu \in \mathcal{M}_{+}(\partial \Omega)} \gamma_{u}(\mu)(\mathcal{O}) \leq M .
$$


REMARK 2.6. The reverse implication

$$
\nu(\mathcal{O})<\infty \Longrightarrow \int_{\mathcal{K}_{t}} u(y) d S_{t} \leq M, \quad \forall t \in\left(0, \beta_{0}\right],
$$

for any compact subset $K \subset \mathcal{O}$, where $M=M(K)>0$, may not hold in the case of general inequalities. However, $g \in \mathcal{H} \mathcal{G}_{0}$ with $\alpha=0$, that is $g$ satisfies $(0.14)$, or if

$$
g(x, r) \leq \rho_{\partial \Omega}^{\alpha}(x) r^{q}=0, \quad \text { in } \Omega \times \mathbb{R}_{+},
$$

with $\alpha>-2$, and $1<q<(N+1+\alpha) /(N-1)$, such a result is still valid. Under both assumptions the proof is much intricated : in the first one it is given in next section, and in the second one, in [27]. In both cases the proof is settled on the notion of stability from inside approximations of the Dirichlet problems (0.4) which means that if a sequence of measures $\lambda_{n} \in \mathfrak{M}_{+}(\Omega)$ converges weakly to a measure $\mu \in \mathfrak{M}_{+}(\partial \Omega)$ the solutions $v_{n}$ of the semilinear equation with forcing term

$$
\begin{aligned}
-\Delta v_{n}+g\left(x, v_{n}\right)=\lambda_{n} & \text { in } \Omega, \\
v_{n}=0 & \text { on } \partial \Omega,
\end{aligned}
$$

converges to $u_{\mu}$ locally uniformly in $\Omega$.

The real number $\gamma_{u}(a)$ plays an important role in the study of the boundary behavior of $u$ at $a$. If $a \in \partial \Omega$, we denote by $\mathcal{N}_{a}$ the set of relatively open neighborhoods of $a$ in $\partial \Omega$.

Definition 2.4. We define by $\mathcal{A}(u)$ the set of atoms of $u$,

$$
\mathcal{A}(u)=\left\{a \in \partial \Omega: \gamma_{u}(a)>0\right\}
$$

by $\mathcal{S}(u)$ the singular set of $u$,

$$
\mathcal{S}(u)=\left\{a \in \mathcal{A}: \forall N_{a} \in \mathcal{N}_{a}, \sum_{\omega \in N_{a}} \gamma_{u}(\omega)=\infty\right\},
$$

the symbol $\sum$ being taken in the sense of summable family, and by $\mathcal{R}(u)$ the regular set of $u$,

$$
\mathcal{R}(u)=\partial \Omega \backslash \mathcal{S}(u)=\left\{a \in \mathcal{A}: \exists N_{a} \in \mathcal{N}_{a}, \sum_{\omega \in N_{a}} \gamma_{u}(\omega)<\infty\right\}
$$

The set $\mathcal{S}(u)$ is closed and $\mathcal{R}(u)$ relatively open. Moreover, if $a \in \mathcal{R}(u)$, there exists a relatively open neighborhood $N_{a} \in \mathcal{N}_{a}$ such that $\mathcal{A}(u) \cap N_{a}$ is at most countable.

The next result complements Propositions 2.7 and 2.8 
THEOREM 2.1. Assume the assumptions of Proposition 2.2 are fulfilled, $u$ is a nonnegative solution of (2.6) with boundary trace $v$, and $\mathcal{O}$ is a relatively open subset of $\partial \Omega$ such that $v(\mathcal{O})<\infty$. Then

$$
\sum_{a \in \mathcal{O}} \tilde{\gamma}_{u}(a)<\infty
$$

If we assume moreover that $g \in \mathcal{H} \mathcal{G}_{0}$, then, for any $\omega \in \partial \Omega$, there holds

$$
v(\omega)=\tilde{\gamma}_{u}(\omega)
$$

and the measure $\chi_{\mathcal{O}} v-\sum_{\omega \in \mathcal{O}} \tilde{\gamma}_{u}(\omega) \delta_{\omega}$ has no atom.

Proof. Let $K$ be a finite subset of $\mathcal{R}(u) \cap \mathcal{O}$ and put $\mu_{K}=\sum_{a \in K} \delta_{a}$. Then for any $\lambda>0$

$$
\sum_{a \in K} \tilde{\gamma}_{u}(a, \lambda) \leq \gamma_{u}\left(\lambda \mu_{K}\right)(\mathcal{O}) \leq v(\mathcal{O})
$$

Therefore the following family $\left\{\tilde{\gamma}_{u}(a)\right\}_{a \in \mathcal{O}}$ is summable, and

$$
\sum_{a \in \mathcal{O}} \tilde{\gamma}_{u}(a) \leq v(\mathcal{O})
$$

For the next statement, for any $\lambda>0$ and $\omega \in \partial \Omega$, there holds

$$
v(\omega) \geq \tilde{\gamma}_{u}(\omega, \lambda) \Longrightarrow v(\omega) \geq \tilde{\gamma}_{u}(\omega)
$$

Conversely, for any relatively open neighborhood of $\omega, \mathcal{O}_{\omega}$, there exists a sequence of Radon measures $\mu_{n} \in \mathfrak{M}_{+}(\partial \Omega)$ such that

$$
\int_{\mathcal{O}_{\omega}} d \mu_{n} \uparrow \nu\left(\mathcal{O}_{\omega}\right), \quad \text { as } n \rightarrow \infty .
$$

If we assume that $v(\omega)=\infty$, we know from Proposition 2.7 that $\tilde{\gamma}_{u}(\omega)=\infty$. Thus we assume $v(\omega)<\infty$. For $\epsilon>0$, there exists $\mu_{\epsilon} \in \mathfrak{M}_{+}(\partial \Omega)$ such that

$$
\gamma_{u}\left(\mu_{\epsilon}\right)(\omega) \leq v(\omega) \leq \gamma_{u}\left(\mu_{\epsilon}\right)(\omega)+\epsilon,
$$

and there exists $\eta_{0}>0$ such that $0<\eta \leq \eta_{0}$ implies

$$
\int_{\Gamma_{\eta}(\omega)} d \gamma_{u}\left(\mu_{\epsilon}\right)-\epsilon \leq v(\omega) \leq \int_{\Gamma_{\eta}(\omega)} d \gamma_{u}\left(\mu_{\epsilon}\right)+\epsilon,
$$

where $\Gamma_{\eta}(\omega)=B_{\eta}(\omega) \cap \partial \Omega$, which yields to

$$
\left|\int_{\Gamma_{\eta}(\omega)} d \gamma_{u}\left(\mu_{\epsilon, \eta}\right)-v(\omega)\right| \leq 2 \epsilon .
$$


If we take $\epsilon=1 / n$, then $\eta_{0}=\eta_{0}(n) \rightarrow 0$ and $\chi_{\Gamma_{\eta}(\omega)} \gamma_{u}\left(\mu_{\epsilon}\right) \rightarrow \nu(\omega) \delta_{\omega}$ as $n \rightarrow \infty$. But

$$
u_{\Gamma_{\Gamma_{\eta}(\omega)} \gamma_{u}\left(\mu_{\epsilon}\right)} \leq u_{\gamma_{u}\left(\mu_{\epsilon}\right)} \leq w_{\gamma_{u}\left(\mu_{\epsilon}\right)} \leq u .
$$

Letting $n \rightarrow \infty$ and using the fact that $u_{\chi_{\Gamma_{\eta}(\omega)} \gamma_{u}\left(\mu_{\epsilon}\right)} \rightarrow u_{\nu(\omega) \delta_{\omega}}$ implies

$$
u_{v(\omega) \delta_{\omega}} \leq u
$$

Therefore

$$
u_{v(\omega) \delta_{\omega}}=\min \left\{u, u_{\nu(\omega) \delta_{\omega}}\right\}=w_{\nu(\omega) \delta_{\omega}}=u_{\tilde{\gamma}_{u}(\omega, v(\omega)) \delta_{\omega}} \leq u_{\tilde{\gamma}_{u}(\omega) \delta_{\omega}} .
$$

This implies $v(\omega) \leq \gamma_{u}(\omega)$ and the equality follows. Consequently $\chi_{\mathcal{O}} v-$ $\sum_{\omega \in \mathcal{O}} \tilde{\gamma}_{u}(\omega) \delta_{\omega}$ has no atom.

\section{3. - Pointwise boundary behaviour of solutions of general inequalities}

In this section, we give a precise description of the behaviour of a solution $u$ of (2.6) near an atom of its extended boundary trace. We say that the coordinates are proper at $a=\left(a_{1}, \ldots, a_{N}\right) \in \partial \Omega$ relatively to $\Omega$ if the plane $x_{1}-a_{1}=0$ is tangent to $\partial \Omega$ at $a$, and that the inward pointing vector to $\partial \Omega$ is the direction $x_{1}-a_{1}>0$.

Definition 3.1. Let $(E, \Sigma, \mu)$ be a measured space, where $\Sigma$ is $\sigma$-algebra of subsets of $E$ and $\mu$ a positive and $\sigma$-additive measure with finite mass. We recall that a set of $\mu$-measurable functions $x \mapsto \psi_{r}(x)(r>0)$, defined over $E$ converges in measure to $\psi$ when $r \rightarrow 0$, if for any $\varepsilon>0$ there holds

$$
\lim _{r \rightarrow 0} \mu\left\{x \in E:\left|\psi_{r}(x)-\psi(x)\right|>\varepsilon\right\}=0 .
$$

The functions $\psi_{r}$ converges in measure to $\infty$, if for any $k>0$,

$$
\lim _{r \rightarrow 0} \mu\left\{x \in E: \psi_{r}(x) \leq k\right\}=0 .
$$

The convergence is equivalent to the following statement: from any sequence $\left\{r_{n}\right\}$ converging to 0 one can extract a subsequence $\left\{r_{n_{k}}\right\}$ such that $\psi_{r_{n_{k}}}$ converges to $\psi($ or $\infty), \mu$-a.e. in $E$. 
THEOREM 3.1. Assume $g \in \mathcal{G}_{0}$ is positively subcritical, $u$ is a nonnegative solution of (2.6) and $a \in \partial \Omega$. If the coordinates are proper at a relatively to $\Omega$, the following alternative holds. Either

(i) $\tilde{\gamma}_{u}(a)$ is finite and the following convergence holds

$$
\lim _{\substack{x \rightarrow a \\\left(x_{1}-a_{1}\right) /|x-a| \rightarrow \eta_{1}}}|x-a|^{N-1} u(x)-C(N) \tilde{\gamma}_{u}(a) \eta_{1}=0,
$$

in measure on $S_{+}^{N-1}$,

or

(ii) $\tilde{\gamma}_{u}(a)$ is infinite and

$$
\lim _{r \rightarrow 0}|x-a|^{N-1} u(x)=\infty
$$

in measure on $S_{+}^{N-1}$.

For $s>0$, put $\Omega \cap B_{s}(a)=\Omega_{s}(a), \Omega_{s}^{c}(a)=\Omega \backslash \bar{\Omega}_{s}(a)$ and $\partial B_{s}(a) \cap$ $\Omega=\Gamma_{s}^{\Omega}(a)$. The next series of results deals with the boundary behaviour of the Green potential of a weighted integrable function. In the flat case where $\Omega=\mathbb{R}_{+}^{N}=\left\{x=\left(x_{1}, \ldots, x_{N}\right): x_{1}>0\right\}$ the computation can be explicited

Lemma 3.1. Let $\Omega=\mathbb{R}_{+}^{N}, N \geq 2, \Phi \in L^{1}\left(\mathbb{R}_{+}^{N} ; x_{1} d x\right)$ and $v=\mathbb{G}_{\Phi}$. Then for any $a \in \partial \mathbb{R}_{+}^{N}$ there holds

$$
\lim _{x \rightarrow a}|x-a|^{-1} \int_{\Gamma_{S}^{\Omega}(a)}|v| x_{1} d S=0 .
$$

Proof. We can assume that $a=0, \Phi \geq 0$, and so is $v$. For $\varepsilon>0$, let $s>0$ such that

$$
\int_{B_{S}(a)} \Phi \rho_{\partial \Omega} d x \leq \varepsilon
$$

Let $(r, \sigma) \in(0,+\infty) \times S^{N-1}$ be the spherical coordinates in $\mathbb{R}^{N}, S_{+}^{N-1}=$ $S^{N-1} \cap \mathbb{R}_{+}^{N}$ and $v(x)=v(r, \sigma)$, then

$$
-\partial_{r r} v-\frac{N-1}{r} \partial_{r} v-\frac{1}{r^{2}} \Delta_{\sigma} v=\Phi,
$$

where $\Delta_{\sigma}$ is the Laplace Beltami operator on $S^{N-1}$. Since $N-1$ is the first eigenvalue of $-\Delta_{\sigma}$ in $W_{0}^{1,2}\left(S_{+}^{N-1}\right)$ and $\phi_{1}(\sigma)=\left.x_{1}\right|_{S_{+}^{N-1}}$, the first eigenfunction, there holds

$$
-\bar{v}_{r r}-\frac{N-1}{r} \bar{v}_{r}+\frac{N-1}{r^{2}} \bar{v}=\bar{\Phi},
$$

where

$$
\bar{v}(r)=\int_{S_{+}^{N-1}} v(r, \sigma) \phi_{1}(\sigma) d \sigma \quad \text { and } \bar{\Phi}(r)=\int_{S_{+}^{N-1}} \Phi(r, \sigma) \phi_{1}(\sigma) d \sigma .
$$


Integrating the above differential equation yields to

$$
\bar{v}(r)=\alpha r^{1-N}+\beta r-\frac{r}{N} \int_{0}^{r} \bar{\Phi}(s) d s+\frac{r^{1-N}}{N} \int_{0}^{r} \bar{\Phi}(s) s^{N} d s,
$$

for some constants $\alpha \geq 0$ and $\beta$. But $\alpha=0$ otherwhile $v$ would be bounded from below by $\alpha C(N) P(x, 0)$. This is impossible because $v$ admits the zero measure for trace on the boundary. Thus

$$
\limsup _{r \rightarrow 0} r^{N-1} \bar{v}(r)=0,
$$

since

$$
\int_{0}^{r} \bar{\Phi}(s) s^{N} d s=\int_{B_{r}(0)} \Phi(x) x_{1} d x
$$

and the result follows.

This result is immediately extendable for any domain which can be deduced by a conformal transformation from a half space.

LEMMA 3.2. Let $\Omega$ be a ball or the complementary of a ball, $\Phi \in L^{1}\left(\Omega ; \rho_{\partial \Omega} d x\right)$ and $v=\mathbb{G}_{\Phi}$. Then for any $a \in \partial \Omega$ there holds

$$
\lim _{x \rightarrow a}|x-a|^{-1} \int_{\Gamma_{S}^{\Omega}(a)}|v| \rho_{\partial \Omega} d S=0 .
$$

In the next lemma we prove that this result is actually always valid. Our proof involves Marcinkiewicz space estimates on the Green potential of a weighted integrable function. The following estimates, similar to (1.22), (1.23), can be found in [4, Theorem 2.6]

$$
\begin{aligned}
\left\|\mathbb{G}_{\Phi}\right\|_{M^{(N+1) /(N-1)\left(\Omega ; \rho_{\partial \Omega} d x\right)}} & \leq K\|\Phi\|_{L^{1}\left(\Omega ; \rho_{\partial \Omega} d x\right)}, \\
\left\|\mathbb{G}_{\Phi}\right\|_{M^{N /(N-1)}(\Omega)} & \leq K\|\Phi\|_{L^{1}\left(\Omega ; \rho_{\partial \Omega} d x\right)} .
\end{aligned}
$$

Actually (3.5) is obtained in [4] only in the case $N \geq 3$, but an easy adaptation of the proof fills the gap. holds

Lemma 3.3. Let $N \geq 2, \Phi \in L^{1}\left(\Omega ; \rho_{\partial \Omega} d x\right)$ and $v=\mathbb{G}_{\Phi}$. If $a \in \partial \Omega$, there

$$
\lim _{r \rightarrow 0} r^{-1} \int_{\Gamma_{S}^{\Omega}(a)}|v| \rho_{\partial \Omega} d S=0 .
$$

Proof. We still assume $\Phi \geq 0$. For $\varepsilon>0$ let $s>0$ be such that

$$
\int_{\Omega_{S}(a)} \Phi \rho_{\partial \Omega} d x \leq \varepsilon
$$


Set $\Phi_{s}=\chi_{\Omega_{s}^{c}(a)}$ and $v_{s}=\mathbb{G}_{\Phi_{s}}$. Since $v_{s}$ is harmonic in $\Omega_{s}(a)$, with zero trace on $\partial \Omega \cap B_{s}(a)$, it is continuous in a neighborhood of $a$ and

$$
\lim _{r \rightarrow 0} r^{-1} \int_{\Gamma_{S}^{\Omega}(a)}|v|_{S} \rho_{\partial \Omega} d S=0
$$

Thus there is no loss of generality in assuming that $\Phi$ has support in $\Omega_{s}(a)$ and

$$
\|\Phi\|_{L^{1}\left(\Omega ; \rho_{\partial \Omega} d x\right)} \leq \varepsilon .
$$

For any $r>0$ and any $\zeta \in C_{c}^{1,1}\left(\bar{\Omega}_{r}(a)\right)$, there holds

$$
-\int_{\Omega_{r}(a)} v \Delta \zeta d x+\int_{\Gamma_{r}^{\Omega_{(}(a)}} \frac{\partial \zeta}{\partial \mathbf{n}} v d S=\int_{\Omega_{r}(a)} \Phi \zeta d x .
$$

This can be established in assuming first that $\Phi=\Phi_{n}$ is regular, and then by density in $L^{1}\left(\Omega ; \rho_{\partial \Omega} d x\right)$. By translation it can be assumed that $a=0$. We set $\zeta(x)=|x| \eta_{r}(x)$, where $\eta_{r}$ is the first eigenfunction of $-\Delta$ in $W_{0}^{1,2}\left(\Omega_{r}(0)\right)$, and let $\lambda_{r}$ be the corresponding eigenvalue. Notice that $r^{2} \lambda_{r} \approx \lambda_{1}$ where $\lambda_{1}$ is the first eigenvalue of the operator

$$
\ell \mapsto-\ell^{\prime \prime}-\frac{N-1}{s} \ell^{\prime}+\frac{N-1}{s^{2}} \ell \text { on }(0,1),
$$

subject to the limit conditions $\ell^{\prime}(0)=0, \ell(1)=0$ (thus the corresponding eigenfuction for (3.9) is a Bessel function, say $B_{1}$, and $\eta_{r}(x) \equiv B_{1}(x / r) x_{1}$ as $r \rightarrow 0$ ). Then (3.8) becomes

$$
\begin{aligned}
& \lambda_{r} \int_{\Omega_{r}(0)} v|x| \eta_{r}(x) d x-\int_{\Omega_{r}(0)} v|x|^{-1}\left\langle x . \nabla \eta_{r}\right\rangle d x \\
& \quad=(N-1) \int_{\Omega_{r}(0)} \eta_{r} u d x-\int_{\Gamma_{r}^{\Omega}(0)}\left(r+\left\langle x . \nabla \eta_{r}\right\rangle\right) v d S+\int_{\Omega_{r}(0)} \Phi|x| \eta_{r} d x
\end{aligned}
$$

Thus

$$
\begin{aligned}
& \limsup _{r \rightarrow 0} \int_{\Gamma_{r}^{\Omega}(0)}\left\langle x . \nabla \eta_{r}\right\rangle v d S \leq \limsup _{r \rightarrow 0} \lambda_{r} \int_{\Omega_{r}(0)} v|x| \eta_{r}(x) d x \\
& \quad+\underset{r \rightarrow 0}{\limsup } \int_{\Omega_{r}(0)} v|x|^{-1}\left|\left\langle x . \nabla \eta_{r}\right\rangle\right| d x .
\end{aligned}
$$

But

$$
|x| \eta_{r}(x) \leq C \rho_{\partial \Omega}(x)\left|\left\langle x . \nabla \eta_{r}\right\rangle\right| \leq C|x| / r,
$$

and more precisely,

$$
\left.\lim _{r \rightarrow 0}\left\langle x . \nabla \eta_{r}\right\rangle\right|_{|x|=r}=\phi_{1}(\sigma)=x_{1} /|x| .
$$


Then

$$
\begin{aligned}
\int_{\Omega_{r}(0)} v|x| \eta_{r}(x) d x & \leq C \int_{\Omega_{r}(0)} \rho_{\partial \Omega}(x) v d x \\
& \leq C\|v\|_{\left.M^{(N+1) /(N-1)\left(\Omega ; \rho_{\partial \Omega}\right.} d x\right)}\left(\int_{\Omega_{r}(0)} \rho_{\partial \Omega} d x\right)^{2 /(N+1)}, \\
& \leq C C^{\prime} r^{2}\|\Phi\|_{L^{1}\left(\Omega ; \rho_{\partial \Omega} d x\right)}
\end{aligned}
$$

and

$$
\begin{aligned}
\int_{\Omega_{r}(0)} v|x|^{-1}\left|\left\langle x . \nabla \eta_{r}\right\rangle\right| d x & \leq C r^{-1} \int_{\Omega_{r}(0)} v d x \\
& \leq C r^{-1}\|v\|_{M^{N /(N-1)(\Omega)}}\left|\Omega_{r}(0)\right|^{1 / N} \\
& \leq C C^{\prime}\|\Phi\|_{L^{1}\left(\Omega ; \rho_{\partial \Omega} d x\right)} .
\end{aligned}
$$

Combining (3.10), (3.11) and (3.12) yields to

$$
\limsup _{r \rightarrow 0} r^{-1} \int_{\Gamma_{r}^{\Omega}(0)} v \rho_{\partial \Omega} d S \leq C^{\prime \prime}\|\Phi\|_{L^{1}\left(\Omega ; \rho_{\partial \Omega} d x\right)} \leq C \varepsilon,
$$

which ends the proof since $\varepsilon$ is arbitrary.

LEMMA 3.4. Assume the assumptions of Theorem 3.1 are fulfilled, $u$ is a nonnegative solution of (2.6), $\lambda>0$ and $a \in \partial \Omega$. If the coordinates are proper at $a$ relatively to $\Omega$, then for any $q \in[1, \infty)$,

$$
\lim _{r \rightarrow 0} r^{N-2}\left(\int_{\Gamma_{r}^{\Omega_{(}(a)}}\left|u_{\lambda \delta_{a}}(y)-\lambda P(y, a)\right|^{q} \rho_{\partial \Omega}(y) d S\right)^{1 / q}=0 .
$$

ProOF. Recall that $g\left(., u_{\lambda \delta_{a}}\right) \in L^{1}\left(\Omega ; \rho_{\partial \Omega} d x\right)$, we put $v=\mathbb{G}_{g\left(., u_{\lambda \delta_{a}}\right)}$. Since $u_{\lambda \delta_{a}}=\lambda P(., a)-v$, it follows from Lemma 3.2

$$
\lim _{r \rightarrow 0} r^{-1} \int_{\Gamma_{r}^{\Omega}(a)}\left|u_{\lambda \delta_{a}}(y)-\lambda P(y, a)\right| \rho_{\partial \Omega}(y) d S=0 .
$$

Since $0 \leq u_{\lambda \delta_{a}} \leq \lambda P(., a)$

$$
y \mapsto r^{N-2} \sup _{y \in \Gamma_{r}^{\Omega}(a)}\left|u_{\lambda \delta_{a}}(y)-\lambda P(y, a)\right| \rho_{\partial \Omega}(y)
$$

is bounded independently of $r$. Thus the result follows by Hölder's inequality.

Under a pointwise growth estimate on $g$ the convergence of $u_{\lambda \delta_{a}}$ is much more precise. 
Lemma 3.5. Let the conditions of Theorem 3.1 be fulfilled. Assume also that there exists $\varepsilon_{0}>0$ such that the mapping $(k, x) \mapsto k^{N+1} g\left(k(x-a)+a, k^{1-N}\right)$ remains bounded for $(k, x) \in\left(0, \varepsilon_{0}\right] \times\left\{x \in a+k^{-1}(\Omega-a): 1-\varepsilon_{0} \leq|x| \leq 1+\varepsilon_{0}\right\}$. Then for any $\eta_{1}>0$,

$$
\lim _{\substack{x \rightarrow a \\(x-a) /|x-a| \rightarrow \eta_{1}}}|x-a|^{N-1} u_{\lambda \delta_{a}}(x)=\lambda C(N) \eta_{1}
$$

for some constant $C(N)>0$. Moreover, for any $\eta>0$, the convergence is uniform in the cone $\eta_{1} \geq \eta$.

Proof. We can assume $a=0$ and set $u_{k}(x)=k^{N-1} u(k x)$. Then

$$
\Delta u_{k}(x)=k^{N+1} g(k x, u(k x)) .
$$

Since $0 \leq u \leq \lambda P(x, 0) \leq \lambda C(N)|x|^{1-N}, u_{k}(x)$ and $(k, x) \mapsto k^{N+1} g(k x, u(k x))$ remains bounded for $(k, x) \in\left(0, \varepsilon_{0}\right] \times\left\{x \in k^{-1} \Omega: 1-\varepsilon_{0} \leq|x| \leq 1+\varepsilon_{0}\right\}$. Thus $\left\{u_{k}\right\}$ is relatively compact in $\left\{x \in k^{-1} \Omega: 1-\varepsilon_{0} / 2 \leq|x| \leq 1+\varepsilon_{0} / 2\right\}$, and there exist a sequence $\left\{k_{n}\right\}$ and some function $\zeta \in C^{1}\left(\overline{\mathbb{R}}_{+}^{N} \cap\left(\bar{B}_{1+\varepsilon_{0}}(0) \backslash B_{1-\varepsilon_{0}}(0)\right)\right)$ such that $u_{k_{n}} \rightarrow \zeta$ and $\nabla u_{k_{n}} \rightarrow \nabla \zeta$ uniformly on $\mathbb{R}_{+}^{N} \cap\left(\bar{B}_{1+\varepsilon_{0} / 2}(0) \backslash B_{1-\varepsilon_{0} / 2}(0)\right)$. Putting $|x|=1$, it implies

$$
\lim _{k_{n} \rightarrow 0} k_{n}^{N-1} u\left(k_{n}, \sigma\right)=\zeta(\sigma)
$$

uniformly on any compact subset of $S_{+}^{N-1}$. Since $P(x, 0)=P(r, \sigma, 0)=$ $C(N) r^{1-N} \phi_{1}(\sigma)$, with $\phi_{1}(\sigma)=\left.x_{1}\right|_{S_{+}^{N-1}}$, the relation (3.15) yields to $\zeta(\sigma)=$ $C(N) \lambda \phi_{1}(\sigma)$, and finally

$$
\lim _{k \rightarrow 0} k_{n}^{N-1} u(k, .)=C(N) \lambda \phi_{1}(.) .
$$

When $u_{\lambda \delta_{a}}$ is replaced by $w_{\lambda \delta_{a}}$, the convergence is comparable to the one of Lemma 3.4.

LEMMA 3.6. Let the assumption of Theorem 3.1 be fulfilled. If $\lambda>0, a \in \partial \Omega$ and the coordinates are proper at a relatively to $\Omega$, there holds

$$
\lim _{r \rightarrow 0} r^{N-2}\left(\int_{\Gamma_{r}^{\Omega}(a)}\left|w_{\lambda \delta_{a}(y)}-\gamma(a, \lambda) P(y, a)\right|^{q} \rho_{\partial \Omega}(y) d S\right)^{1 / q}=0,
$$

for any $1 \leq q<\infty$. 
PROOF. Since $\Delta w_{\lambda \delta_{a}}$ and $g\left(., w_{\lambda \delta_{a}}\right)$ belong to $L^{1}\left(\Omega ; \rho_{\partial \Omega} d x\right)$, there exists $\Phi \in L^{1}\left(\Omega ; \rho_{\partial \Omega} d x\right)$ such that

$$
\begin{aligned}
& -\Delta w_{\lambda \delta_{a}}=\Phi \quad \text { in } \Omega \text {, } \\
& w_{\lambda \delta_{a}}=\gamma(a, \lambda) \delta_{0} \text { on } \partial \Omega \text {. }
\end{aligned}
$$

Then $w_{\lambda \delta_{a}}=\mathbb{G}_{\Phi}+\gamma(a, \lambda) P(., a)$ and

$$
\left|w_{\lambda \delta_{a}}-\gamma(a, \lambda) P(., a)\right| \leq \mathbb{G}_{|\Phi|} .
$$

By Lemma 3.3

$$
\lim _{r \rightarrow 0} r^{N-2} \int_{\Gamma_{r}^{\Omega}(a)}\left|\mathbb{G}_{\Phi}(y)\right| d S=0,
$$

thus

$$
\lim _{r \rightarrow 0} r^{N-2} \int_{\Gamma_{r}^{\Omega}(a)}\left|w_{\lambda \delta_{a}}(y)-\gamma(a, \lambda) P(y, a)\right| \rho_{\partial \Omega}(y) d S=0 .
$$

Since $0 \leq w_{\lambda \delta_{a}} \leq \lambda P(., a)$, and $r^{N-2} \rho_{\partial \Omega} P(., a)$ is bounded on $\Gamma_{r}^{\Omega}(a)$, (3.17) follows.

Proof OF THEOREM 3.1. Up to a translation, we can assume that $a=0$. We can assume that $S_{+}^{N-1}$ is the intersection of the unit sphere with the half space $\left\{x_{1}>0\right\}$ and $\partial S_{+}^{N-1}$ the intersection of $S^{N-1}$ with the hyperplane $\left\{x_{1}=0\right\}$. Thus $\phi_{1}$, the first eigenvalue of the Laplace-Beltrami operator $-\Delta_{\sigma}$ in $W_{0}^{1,2}\left(S_{+}^{N-1}\right)$ is the restriction to $S^{N-1}$ of the coordinate function $x \mapsto x_{1}$, and the corresponding eigenvalue is $N-1$. We normalize by $\max \phi_{1}=1$.

CASE 1. $\gamma(a)<\infty$. For $\lambda>\gamma(a)$ the following convergences hold in $L^{q}\left(S_{+}^{N-1}\right)$ for $1 \leq q<\infty$ :

$$
\lim _{r \rightarrow 0} r^{N-1} w_{\lambda \delta_{a}}(r, .)=C(N) \gamma(a, \lambda) \phi_{1}
$$

by Lemma 3.6, and

$$
\lim _{r \rightarrow 0} r^{N-1} u_{\lambda \delta_{a}}(r, \sigma)=C(N) \lambda \phi_{1}(\sigma)
$$

by Lemma 3.4. If $\left\{r_{n}\right\}$ is some sequence converging to 0 , there exists a subsequence $\left\{r_{n_{k}}\right\}$ such that

$$
\lim _{r_{n_{k}} \rightarrow 0} r_{n_{k}}^{N-1} w_{\lambda \delta_{a}}\left(r_{n_{k}}, \sigma\right)=C(N) \tilde{\gamma}_{u}(a, \lambda) \phi_{1}(\sigma),
$$

and

$$
\lim _{r_{n_{k}} \rightarrow 0} r_{n_{k}}^{N-1} u_{\lambda \delta_{a}}\left(r_{n_{k}}, \sigma\right)=C(N) \lambda \phi_{1}(\sigma),
$$


for almost all $\sigma \in S_{+}^{N-1}$. Therefore for almost all $\sigma \in S_{+}^{N-1}$, there exists $n_{k_{0}}$ such that for $n_{k} \geq n_{k_{O}}, w_{\lambda \delta_{a}}\left(r_{n_{k}}, \sigma\right)=u\left(r_{n_{k}}, \sigma\right)$. Consequently there holds

$$
\lim _{r \rightarrow 0} r^{N-1} u\left(r_{n_{k}}, \sigma\right)=C(N) \tilde{\gamma}_{u}(a, \lambda) \phi_{1}(\sigma),
$$

for almost all $\sigma \in S_{+}^{N-1}$. Let $\theta>\lambda$. It follows from Lemma 3.5 applied to the $w_{\theta \delta_{a}}\left(r_{n_{k}},.\right)$ and the previous argument, that, up to some subsequence $r_{n_{k_{\ell}}}$,

$$
\lim _{r \rightarrow 0} r^{N-1} u\left(r_{n_{k_{\ell}}}, \sigma\right)=C(N) \tilde{\gamma}_{u}(a, \theta) \phi_{1}(\sigma)
$$

almost everywhere. Therefore $\tilde{\gamma}_{u}(a, \theta)=\tilde{\gamma}_{u}(a, \lambda)=\tilde{\gamma}_{u}(a)$. This infers (i).

CASe 2. $\tilde{\gamma}_{u}(a)=\infty$. From Lemma 2.1,

$$
\min \left(u, u_{\infty, a}\right) \geq u_{\infty, a} \Longrightarrow u \geq u_{\infty, a}>u_{\lambda \delta_{a}}, \quad \forall \lambda>0 .
$$

By Lemma 3.4, for any $\varepsilon>0$,

$$
\liminf _{\substack{x \rightarrow a \\\left(x_{1}-a_{1}\right) /|x-a| \rightarrow \eta_{1} \\ \eta_{1} \geq \varepsilon}}|x-a|^{N-1} u(x) \geq C(N) \lambda \varepsilon .
$$

Since $\lambda$ is arbitrary, (ii) holds.

REMARK 3.1. In the core of the proof in Case 1 we have seen that $\tilde{\gamma}_{u}(\lambda, a)=\tilde{\gamma}_{u}(a)$ for any $\lambda>\gamma(a)$. Actually the same proof gives also $\tilde{\gamma}_{u}(\lambda, a)=\tilde{\gamma}_{u}(a)$ for $\lambda=\tilde{\gamma}_{u}(a)$.

REMARK 3.2. If it is supposed moreover that $(k, x) \mapsto k^{N+1} g(k(x-a)+$ $\left.a, k^{1-N}\right)$ remains bounded for $(k, x) \in\left(0, \varepsilon_{0}\right] \times\left\{x \in a+k^{-1}(\Omega-a): 1-\varepsilon_{0} \leq\right.$ $\left.|x| \leq 1+\varepsilon_{0}\right\}$, assertion (ii) can be replaced by:

or

(ii*) $\tilde{\gamma}_{u}(a)$ is infinite and

$$
\lim _{\substack{x \rightarrow a \\\left(x_{1}-a_{1}\right) /|x-a| \rightarrow \eta_{1}}}|x-a|^{N-1} u(x)=\infty,
$$

uniformly for $\eta_{1}>0$. 


\section{4. - Boundary trace of solutions with uniform absorption}

In this section $\Omega$ is a $C^{2}$ bounded domain in $\mathbb{R}^{N}, g \in \mathcal{G}_{0}$ satisfies a uniform condition with respect to $x$ in the sense that

$$
\begin{aligned}
& 0 \leq|g(x, r)| \leq f(r), \forall(x, r) \in \Omega \times \mathbb{R}_{+}, \\
& \text {with } \int_{0}^{1} f\left(\sigma s^{1-N}\right) s^{N} d s<\infty, \forall \sigma>0,
\end{aligned}
$$

where $f$ is a continuous nondecreasing function defined on $\mathbb{R}_{+}$. The next result provides a precise characterisation of the boundary trace of solutions of inequalities with a uniform absorption in terms of outer regular Borel measures, without introducing the notion of coercivity and the strong barrier property as in [27].

THEOREM 4.1. Assume $g \in \mathcal{G}_{0}$ satisfies (4.1) and $u$ is a nonnegative solution of (2.6) with boundary trace $v$. For any $a \in \partial \Omega$ the following dichotomy occurs. Either,

(i) $v(\mathcal{O})=\infty$ for any $\mathcal{O} \in \mathcal{N}_{a}$. In this case $a \in \mathcal{S}(u)$ and $u \geq u_{\infty, a}$. Consequently

$$
\lim _{t \rightarrow 0} \int_{\mathcal{O}_{t}} u(y) d S_{t}=\infty, \forall \mathcal{O} \in \mathcal{N}_{a}
$$

$\mathrm{Or}$

(ii) there exists $\mathcal{O} \in \mathcal{N}_{a}$ such that $v(\mathcal{O})<\infty$. In this case $a \in \mathcal{R}(u)$ and

$$
\sup _{0<t \leq \beta_{0}} \int_{\mathcal{O}_{t}^{\prime}} u(y) d S_{t}<\infty .
$$

for relatively every open subset $\mathcal{O}^{\prime} \subset \overline{\mathcal{O}^{\prime}} \subset \mathcal{O}$. Furthermore

$$
\lim _{t \rightarrow 0} \int_{\Sigma_{t}} u(y) \phi(\sigma(y))(y) d S_{t}=\int_{\mathcal{R}(u)} \phi(y) d v(y), \quad \forall \phi \in C_{c}(\mathcal{R}(u))
$$

A major point in the proof of the theorem is the following completion of Proposition 2.7 which gives a characterization of the regular part of the extended boundary trace of a solution $u$ based upon a local $L^{1}$ bound.

Proposition 4.1. Assume $g \in \mathcal{G}_{0}$ satisfies (4.1) and $u$ is a nonnegative solution of (2.6) with extended boundary trace $\nu$. Let $\mathcal{O}$ be a relatively open subset of $\partial \Omega$. If $v(\mathcal{O})<\infty$, then for any compact subset $K \subset \mathcal{O}, \int_{K_{t}} u(y) d S_{t}$ remains bounded independently of $t \in\left(0, \beta_{0}\right]$.

We recall some notations introduced in Section 2 : for $0<\beta \leq \beta_{0}$, we put $\Omega_{\beta}^{c}=\Omega \backslash \bar{\Omega}_{\beta}=\left\{x \in \Omega: \rho_{\partial \Omega}(x)>\beta\right\}$, and $\Sigma_{\beta}=\partial \Omega_{\beta}=\partial \Omega_{\beta}^{c}$. The next result which extends Theorem 1.1 deals with the stability of the boundary value problem when the boundary is approximated from inside by a sequence of smooth domains. 
LEMMA 4.1. Let $\mu \in \mathfrak{M}_{+}(\partial \Omega),\left\{\varepsilon_{n}\right\}$ be a sequence of positive numbers converging to $0, \mu_{n} \in L_{+}^{1}\left(\Sigma_{\varepsilon_{n}}\right)$, with corresponding pull-back $\mu_{n}^{\varepsilon_{n}} \in L_{+}^{1}(\partial \Omega)$. If $\mu_{n}^{\varepsilon_{n}} \rightarrow \mu$ in the weak sense of measures, as $n \rightarrow \infty$, then the sequence of solutions $u_{n}=u_{\mu_{n}, \varepsilon_{n}}$ of

$$
\begin{aligned}
-\Delta u_{n}+g\left(x, u_{n}\right) & =0 \quad \text { in } \Omega_{\varepsilon_{n}}^{c}, \\
u_{n} & =\mu_{n} \text { on } \partial \Omega_{\varepsilon_{n}}^{c},
\end{aligned}
$$

converges locally uniformly in $\Omega$ to the solution $u_{\mu}$ of $(0.4)$.

Proof. STEP 1. Since $g$ is continuous on $\bar{\Omega}_{\varepsilon_{n}}^{c} \times \mathbb{R}$, existence of a unique solution to (4.6) follows from Brezis' result. The shred of the proof of the convergence is parallel to Theorem 1.1 and Proposition 1.4. Set $P^{\varepsilon_{n}}$ the Poisson kernel in $\Omega_{\varepsilon_{n}}^{c}$ and $\mathbb{P}_{v}^{\varepsilon_{n}}$ the Poisson potential of any given Radon measure $v$ on $\Sigma_{\varepsilon_{n}}$. Since the mapping $\Pi$ is $C^{2}$ there exists $C_{2}>0$ independent $\varepsilon_{n}$ such that for any $(x, a) \in \Omega_{\varepsilon_{n}}^{c} \times \Sigma_{\varepsilon_{n}}$,

$$
C_{2}^{-1} \rho_{\partial \Omega_{\varepsilon_{n}}^{c}}(x)|x-a|^{-N} \leq P^{\varepsilon_{n}}(x, a) \leq C_{2} \rho_{\partial \Omega_{\varepsilon_{n}}^{c}}(x)|x-a|^{-N},
$$

provided $0 \leq \varepsilon_{n} \leq \beta_{0}$, and $\rho_{\partial \Omega_{\varepsilon_{n}}^{c}}(x)=\rho_{\partial \Omega}(x)-\varepsilon_{n}$. Estimates (1.22), (1.23), (1.24) are valid under the form

$$
\begin{aligned}
& \left\|\mathbb{P}_{\nu}^{\varepsilon_{n}}\right\|_{\left.M^{(N+1) /(N-1)\left(\Omega_{\varepsilon_{n}}^{c} ; \rho_{\partial \Omega_{\varepsilon_{n}}^{c}}\right.} d x\right)} \leq K\|\nu\|_{L^{1}\left(\Sigma_{\varepsilon_{n}}\right)}, \\
& \left\|\mathbb{P}_{\nu}^{\varepsilon_{n}}\right\|_{M^{N /(N-1)}\left(\Omega_{\varepsilon_{n}}^{c}\right)} \leq K\|v\|_{L^{1}\left(\Sigma_{\varepsilon_{n}}\right)}, \\
& \left\|\mathbb{P}_{v}^{\varepsilon_{n}}\right\|_{L^{\infty}\left(\Omega_{r+\varepsilon_{n}}^{c}\right)} \leq K r^{1-N}\|v\|_{L^{1}\left(\Sigma_{\varepsilon_{n}}\right)},
\end{aligned}
$$

Since $0 \leq u_{n} \leq v_{n}=\mathbb{P}_{\mu_{n}}^{\varepsilon_{n}}$, estimates (4.9) and (1.16)-(1.18) and the classical regularity theory for elliptic equations imply that the set of $u_{n}$ is relatively compact in the $C_{\text {loc }}^{1}(\Omega)$-topology, and any cluster point of the sequence $\left\{u_{n}\right\}$ is a solution of (0.1) in $\Omega$. If $\eta \in C_{c}^{1,1}\left(\bar{\Omega}_{\varepsilon_{n}}^{c}\right)$ there holds

$$
\int_{\Omega_{\varepsilon_{n}^{c}}^{c}}\left(-u_{n} \Delta \eta+g\left(., u_{n}\right) \eta\right) d y=-\int_{\Sigma_{n}} \frac{\partial \eta}{\partial \mathbf{n}_{y}} \mu_{n} d S(y)
$$

If $\zeta \in C_{c}^{1,1}(\bar{\Omega})$, with support in $\bar{\Omega}_{\beta_{0}}$, we set

$$
\zeta_{n}(y)=\zeta\left(y+\varepsilon_{n} \mathbf{n}_{y}\right), \quad \forall y \in \bar{\Omega}_{\varepsilon_{n}}^{c} \Longleftrightarrow \zeta(x)=\zeta_{n}\left(x-\varepsilon_{n} \mathbf{n}_{x}\right), \quad \forall x \in \bar{\Omega} .
$$

In relation (4.10) we take $\eta=\zeta_{n}$ and get

$$
\int_{\Omega_{\varepsilon_{n}}^{c}}\left(-u_{n} \Delta \zeta_{n}+g\left(., u_{n}\right) \zeta_{n}\right) d y=-\int_{\Sigma_{n}} \frac{\partial \zeta_{n}}{\partial \mathbf{n}} \mu_{n} d S_{\varepsilon_{n}} .
$$


But the pointing outward normal vector $\mathbf{n}_{y}$ at $y \in \Sigma_{n}$ is the same as the pointing outward normal vector at $y+\varepsilon_{n} \mathbf{n}_{y} y \in \partial \Omega$, therefore

$$
\int_{\Sigma_{n}} \frac{\partial \zeta_{n}}{\partial \mathbf{n}} \mu_{n} d S_{\varepsilon_{n}}=\int_{\partial \Omega} \frac{\partial \zeta}{\partial \mathbf{n}} \mu_{n}^{\varepsilon_{n}} d S
$$

by (2.5). Moreover, if we perform the change of variable $x=y+\varepsilon_{n} \mathbf{n}_{y}$ in (4.11) and denote by $\mathbf{n}_{y}^{j}$ the coordinates of $\mathbf{n}_{y}$, we get

$$
\begin{aligned}
\frac{\partial \zeta_{n}}{\partial y_{i}}(y)= & \sum_{j} \frac{\partial \zeta}{\partial x_{j}}(x)\left(\delta_{i j}+\varepsilon_{n} \frac{\partial \mathbf{n}_{y}^{j}}{\partial y_{i}}\right), \\
\frac{\partial^{2} \zeta_{n}}{\partial y_{i}^{2}}(y)= & \sum_{k, j} \frac{\partial^{2} \zeta}{\partial x_{k} \partial x_{j}}(x)\left(\delta_{i j}+\varepsilon_{n} \frac{\partial \mathbf{n}_{y}^{j}}{\partial y_{i}}\right)\left(\delta_{i k}+\varepsilon_{n} \frac{\partial \mathbf{n}_{y}^{k}}{\partial y_{i}}\right) \\
& +\varepsilon_{n} \sum_{j} \frac{\partial \zeta}{\partial x_{j}}(x)\left(\frac{\partial^{2} \mathbf{n}_{y}^{j}}{\partial y_{i}^{2}}\right), \\
\Delta \zeta_{n}(y)= & \sum_{i} \frac{\partial^{2} \zeta}{\partial x_{i}}(x)\left(1+\varepsilon_{n} \frac{\partial \mathbf{n}_{y}^{i}}{\partial y_{i}}\right)^{2} \\
& +\sum_{\substack{i, j, k \\
k \neq i \text { ouj } \neq i}} \frac{\partial^{2} \zeta}{\partial x_{k} \partial x_{j}}(x)\left(\delta_{i j}+\varepsilon_{n} \frac{\partial \mathbf{n}_{y}^{j}}{\partial y_{i}}\right)\left(\delta_{i k}+\varepsilon_{n} \frac{\partial \mathbf{n}_{y}^{k}}{\partial y_{i}}\right) \\
& +\varepsilon_{n} \sum_{j} \frac{\partial \zeta}{\partial x_{j}}(x) \Delta \mathbf{n}_{y}^{j} .
\end{aligned}
$$

Then

$$
\Delta \zeta_{n}(y)=\Delta \zeta(x)+\varepsilon_{n} \mathcal{L}\left(D \zeta(x), D^{2} \zeta(x)\right)
$$

where $\mathcal{L}\left(D \zeta, D^{2} \zeta\right)$ is a linear second order operator with continuous coefficients. Plugging (4.12) and (4.13) into (4.11) yields

$$
\int_{\Omega}\left(-\tilde{u}_{n}\left(\Delta \zeta+\varepsilon_{n} \mathcal{L}\left(D \zeta, D^{2} \zeta\right)\right)+g_{n}\left(., \tilde{u}_{n}\right) \zeta\right) J d x=-\int_{\partial \Omega} \frac{\partial \zeta}{\partial \mathbf{n}} \mu_{n}^{\varepsilon_{n}} d S
$$

where $\tilde{u}_{n}(x)=u_{n}\left(x-\varepsilon_{n} \mathbf{n}_{x}\right), g_{n}(x, r)=g\left(x-\varepsilon_{n} \mathbf{n}_{x}, r\right)$ and $J(x)=\left|\operatorname{det}\left(I-\varepsilon_{n} D \mathbf{n}_{y}\right)\right|$. STEP 2 From (1.26) and (4.6) there exists $C_{3}>0$ independent of $\varepsilon_{n}$ and $(y, b) \in \Omega_{\varepsilon_{n}}^{c} \times \Sigma_{n}$ such that

$$
C_{3}^{-1} P\left(y+\varepsilon_{n} \mathbf{n}_{y}, b+\varepsilon_{n} \mathbf{n}_{y}\right) \leq P^{\varepsilon_{n}}(y, b) \leq C_{3} P\left(y+\varepsilon_{n} \mathbf{n}_{y}, b+\varepsilon_{n} \mathbf{n}_{y}\right),
$$


provided $\varepsilon_{n} \leq \beta_{0}$. Because $0 \leq u_{n}(y) \leq \mathbb{P}_{\mu_{n}}^{\varepsilon_{n}}$, the above inequality and the Poisson representation formula imply

$$
0 \leq \tilde{u}_{n} \leq C_{3} \mathbb{P}_{\mu_{n}^{\varepsilon_{n}}}
$$

in $\bar{\Omega}_{\beta_{0}-\varepsilon_{n}}$. Jointly with (1.23) it implies that $\left\{\tilde{u}_{n}\right\}$ is uniformly integrable in $\Omega_{\beta_{0}-\varepsilon_{n}}$, and thus in $\Omega$.

STEP 3 From (4.1), (4.16),

$$
0 \leq g_{n}\left(x, \tilde{u}_{n}\right)(x) \leq f\left(C_{3} \mathbb{P}_{\mu_{n}} \varepsilon_{n}(x)\right),
$$

for any $x \in \Omega_{\beta_{0}-\varepsilon_{n}}$. For $\lambda \geq 0$, put $\Gamma_{\lambda}=\{x \in \Omega: P(x, a) \geq \lambda\}$ and

$$
\beta_{\varepsilon_{n}}(\lambda)=\int_{\Gamma_{\lambda}} \rho_{\partial \Omega} d x
$$

Then (see Step 2 in the proof of Theorem 1.1),

$$
\beta_{\varepsilon_{n}}(\lambda) \leq C_{2} \int_{0}^{\left(C_{1} / \lambda\right)^{1 /(N-1)}} s^{N} d s \leq \frac{C_{2}}{N+1}\left(\frac{C_{1}}{\lambda}\right)^{(N+1) /(N-1)}
$$

It follows from (4.17) that for any Borel set $G \subset \Omega$,

$$
\int_{G} g_{n}\left(., \tilde{u}_{n}\right) \rho_{\partial \Omega} d x \leq \int_{G} f\left(C_{3} \mathbb{P}_{\mu_{n}^{\varepsilon n}}\right) \rho_{\partial \Omega} d x .
$$

In order to estimate the right-hand side of (4.19), we follow the proof of Theorem 1.1. Let $m>0$ and $a \in \partial \Omega$, then

$$
\begin{aligned}
\int_{G} f & (m P(., a)) \rho_{\partial \Omega} d x \\
& \leq f(\lambda) \int_{G} \rho_{\partial \Omega} d x+C_{4} m^{(N+1) /(N-1)} \int_{\lambda}^{\infty} f(s) s^{-2 N /(N-1)} d s .
\end{aligned}
$$

We take $m=m_{n}=\int_{\partial \Omega} d \mu_{n}^{\varepsilon_{n}}$ and we deduce that the $\left\{g_{n}\left(., \tilde{u}_{n}\right)\right\}$ are uniformly integrable, as in the proof of Theorem 1.1-Step 7. If $\tilde{u}_{n_{k}}$ is a subsequence converging in the $C_{\text {loc }}^{1}$ topology to some function $u$, we can pass to the limit in (4.14) and get

$$
\int_{\Omega}(-u \Delta \zeta+g(., u) \zeta) d y=-\int_{\partial \Omega} \frac{\partial \zeta}{\partial \mathbf{n}} d \mu
$$

Because of uniqueness, the whole sequence $\tilde{u}_{n}$ converges to $u$. 
REMARK 4.1. This above stability result of solutions with respect to approximations from inside is no longer valid if the absorption term is truly degenerate, for example if

$$
g(x, r)=\rho_{\partial \Omega}^{\alpha}(x)|u|^{q-1} u,
$$

for some $\alpha>0$ and $q>1$. In that case Problem (0.4) is solvable in $\Omega$ for any Radon measure $\mu$ when $1<q<(N+1+\alpha) /(N-1)$ and is not solvable when $\mu$ is a Dirac mass if $q \geq(N+1+\alpha) /(N-1)$ (see [27]). Therefore, even if the data $\mu_{n}$ are smooth functions on $\Sigma_{\varepsilon_{n}}$, if they concentrate too quickly to a Dirac mass on the boundary, the corresponding solutions $u_{n}$ of (4.6) converge to 0 .

ProOF of Proposition 4.1. We proceed by contradiction in assuming that there exist a compact $K \subset \mathcal{O}$ and a sequence $\left\{\varepsilon_{n}\right\}$ converging to 0 such that

$$
\lim _{n \rightarrow \infty} \int_{K_{\varepsilon_{n}}} u(y) d S_{\varepsilon_{n}}=\infty
$$

Since $K$ is compact, there exist a sequence $\left\{a_{n}\{\subset K\right.$ converging to some $a \in K$ and a sequence $\left\{t_{n}\right\}$ converging to 0 such that

$$
\lim _{n \rightarrow \infty} \int_{K_{\varepsilon_{n}} \cap B_{t_{n}}\left(a_{n}\right)} u(y) d S_{\varepsilon_{n}}=\infty .
$$

For $k>0$, there exists $\ell_{k}>0$ such that

$$
\lim _{n \rightarrow \infty} \int_{K_{\varepsilon_{n}} \cap B_{t_{n}}\left(a_{n}\right)} \min \left\{\ell_{k}, u(y)\right\} d S_{\varepsilon_{n}}=k
$$

and $\ell_{k} \rightarrow 0$ as $n \rightarrow \infty$. We set $\mu_{n}=\min \left\{\ell_{k}, u(y)\right\} \chi_{K_{\varepsilon_{n}} \cap B_{t_{n}}\left(a_{n}\right)}$ and denote by $u_{n}$ the solution of (4.6) in $\Omega_{\varepsilon_{n}}^{c}$ with this specific boundary data. Then

$$
u \geq u_{n} \quad \text { in } \quad \Omega_{\varepsilon_{n}}^{c} .
$$

Since the corresponding measure $\gamma_{u}\left(\mu_{n}\right)=\mu_{n}^{\varepsilon_{n}}$ on $\partial \Omega$ converges to $k \delta_{a}$, and $u_{n}$ converges to $u_{k \delta_{a}}$ by Lemma 4.1 , it leads to $u \geq u_{k \delta_{a}}$ in $\Omega$. Since $k$ is arbitrary,

$$
u \geq u_{\infty, a} \text { in } \Omega
$$

Therefore

$$
v(\mathcal{O}) \geq v(a)=\tilde{\gamma}(a)=\infty,
$$

by Proposition 2.7, a contradiction. 
For any $a \in \partial \Omega$, we recall that $\mathcal{N}_{a}$ is the set of relatively open neighborhoods of $a$ in $\partial \Omega$.

Proof of TheOrem 4.1. Let $a \in \partial \Omega$. If (i) holds, inequality $u \geq u_{\infty, a}$ follows from Proposition 2.7, and

$$
\lim _{t \rightarrow 0} \int_{\mathcal{O}_{t}} u(y) d S_{t} \geq \lim _{t \rightarrow 0} \int_{\mathcal{O}_{t}} u_{\infty, a}(y) d S_{t}=\infty
$$

which is equivalent to $v(\mathcal{O})=\infty$.

Next we assume that (i) does not hold, and there exists $\mathcal{O} \in \mathcal{N}_{a}$ such that $v(\mathcal{O})<\infty$. By Proposition 4.1 , for any compact subset $\mathcal{K} \in \mathcal{N}_{a}$ such that $\overline{\mathcal{K}} \subset \mathcal{O}$, there exists a constant $M_{K}>0$ such that

$$
\int_{\mathcal{K}_{t}} u(y) d S_{t} \leq M_{K}, \quad \text { on }\left(0, \beta_{0}\right]
$$

Let $\mathcal{O}$ be any relatively open subset with compact closure in $\mathcal{R}(u), 0<\beta<\beta_{0}$ and

$$
\mathfrak{O}_{\beta}=\left\{x=\sigma(x)-t \mathbf{n}_{x}: \sigma(x) \in \mathcal{O}, \beta<t<\beta_{0}\right\},
$$

then $u \in L^{1}\left(\mathfrak{O}_{0}\right)$. As in the proof of [27, Lemma 1.8], if $\varphi \in C_{c}^{2}(\mathcal{O}), \varphi>0$, we define a test function which vanishes on $G s_{\beta}$ by

$$
\zeta(x)=\varphi(\sigma(x))\left(\rho_{\partial \Omega}(x)-\beta\right) \quad \forall x \in \mathfrak{O}_{\beta},
$$

and derive that the largest solution $u^{*}$ of $(0.1)$ dominated by $u$ satisfies

$$
\begin{aligned}
\int_{\mathfrak{O}_{\beta}}\left(-u^{*} \Delta \zeta+g\left(x, u^{*}\right) \zeta\right) d x= & \int_{\mathcal{O}_{\beta}} u^{*} \varphi^{\beta} d S_{\beta}-\int_{\mathcal{O}_{\beta_{0}}} u^{*} \varphi^{\beta_{0}} d S_{\beta_{0}} \\
& +\int_{\mathcal{O}_{\beta_{0}}} \frac{\partial u^{*}}{\partial \mathbf{n}} \zeta d S .
\end{aligned}
$$

Therefore $\int_{\mathfrak{O}_{\beta}} g\left(x, u^{*}\right) \zeta d x$ is bounded independently of $\beta$. Letting $\beta \rightarrow 0$ yields to

$$
\int_{\mathfrak{O}_{0}} g\left(x, u^{*}\right) \varphi \rho_{\partial \Omega} d x<\infty
$$

Since $g\left(x, u^{*}\right) \in L^{1}\left(\mathfrak{O}_{0} ; \rho_{\partial \Omega} d x\right)$, [27, Corollary 1.3] implies that there exists a nonnegative Radon measure $\mu_{\mathcal{O}}$ on $\mathcal{O}$ such that

$$
\lim _{t \rightarrow 0} \int_{\mathcal{O}_{t}} \varphi_{t} u^{*}(y) d S_{t}=\int_{\mathcal{O}} \varphi d \mu
$$


for any $\varphi \in C_{c}(\mathcal{O})$. The measure $v$, which is equal to $\mu$ on $\mathcal{R}(u)$, is therefore a regular Borel measure.

Because of (4.3) from any sequence $\left\{\epsilon_{n}\right\}$ converging to 0 one can extract a subsequence, still denoted by $\left\{\epsilon_{n}\right\}$, such that $\left\{u\left(\epsilon_{n},\right) \chi_{\mathcal{R}(u)} d S\right\}$ converges in the weak sense of measures to some $\eta \in \mathfrak{M}_{+}(\mathcal{R}(u))$. We claim that

$$
\eta=\left.v\right|_{\mathcal{R}(u)}=\chi_{\mathcal{R}(u)} \nu .
$$

Since $u \geq u^{*}, \eta \geq\left. v\right|_{\mathcal{R}(u)}$. If $\mathcal{O}$ is any relatively open subset of $\partial \Omega$ with compact closure in $\mathcal{R}(u)$, we put $\mu_{n}=u\left(\epsilon_{n},\right) \chi_{\mathcal{R}(u)} d S$ and denote by $u_{n}$ the solution of (4.6) in $\Omega_{\epsilon_{n}}^{c}$. By Lemma 4.1, $\left\{u_{n}\right\}$ converges locally uniformly in $\Omega$ to the solution $\tilde{u}$ of $(0.4)$ with boundary data $\chi_{\mathcal{O}} \eta$. Since $u \geq u_{n}, u \geq \tilde{u}$ and thus $u^{*} \geq \tilde{u}$. Therefore $\chi_{\mathcal{O}} \eta \leq v_{\mathcal{O}}$. This implies (4.27). Finally, as $\eta$ is independent of the sequence $\left\{\epsilon_{n}\right\}$, it follows that $u(t,.) \chi_{\mathcal{R}(u)} d S$ converges to $\chi_{\mathcal{R}(u)} v$ in the sense of measures, as $t \rightarrow 0$. This ends the proof.

\section{5. - Some examples}

In this section $\Omega$ is a $C^{2}$ bounded domain and we consider absorption terms $g$ which are split under the form

$$
g(x, r)=\tilde{h}(x) \tilde{g}(r)
$$

where both $\tilde{h}$ and $\tilde{g}$ are nonnegative continuous functions defined respectively on $\Omega$ and $\mathbb{R}_{+}$. We assume also that $\tilde{g}$ vanishes at 0 and is nondecreasing, and that $\tilde{h}(x)>0$ in $\Omega$. If the the Keller-Osserman condition is fulfilled, that is there exists some $c \geq 0$ such that

$$
\int_{c}^{\infty} \frac{d s}{\sqrt{G(s)}}<\infty
$$

where $G(s)=\int_{0}^{s} \tilde{g}(t) d t$, then for any compact subset $K \subset \Omega$ there exists a constant $C(K)>0$ such that any nonnegative solution $u$ of

$$
-\Delta u+g(x, u) \leq 0, \quad \text { in } \Omega,
$$

satisfies

$$
u(x) \leq C(K), \quad \forall x \in K
$$

If the Keller-Osserman condition is not satisfied, and $\tilde{h}$ is a positive constant, no such a priori upper bound can exist [31]. If $\tilde{g}(r)=k r$ for some $k>0$ and $g$ is uniformly admissible, it is clear that

$$
\lim _{\lambda \rightarrow \infty} u_{\lambda \delta_{a}}(x)=\infty, \quad \forall a \in \partial \Omega, \forall x \in \Omega
$$


but it appears difficult to find a general condition on $\tilde{g}$ which implies that (5.4) holds. However, it is proved in [13] that if

$$
g(x, r)=r(\ln (r+1))^{\gamma},
$$

with $0<\gamma \leq 2$, this property holds. As a consequence we have the following

COROLlaRY 5.1. Let $u$ be a nonnegative solution of

$$
-\Delta u+u(\ln (u+1))^{\gamma}=0, \quad \text { in } \Omega .
$$

Then the boundary trace of $u$ is a Radon measure $\mu, u(\ln (u+1))^{\gamma} \in L^{1}\left(\Omega ; \rho_{\partial \omega} d x\right)$ and

$$
u=\mathbb{P}_{\mu}-\mathbb{G}\left(u(\ln (u+1))^{\gamma}\right) .
$$

Proof. It is clear that $g(u)=u(\ln (u+1))^{\gamma}$ is uniformly admissible. By Theorem 4.1, $\operatorname{Tr}_{\partial \Omega}^{e}(u)$ is an outer regular Borel measure, which admits no singular part by [13], therefore it is a Radon measure. Thus the nonlinearity is integrable for the measure $\rho_{\partial \omega} d x$, and the representation follows.

REMARK 5.1. If

$$
g(x, r)=r(\ln (r+1))^{\gamma}
$$

with $\gamma>2$, or if

$$
g(x, r)=\rho_{\partial \Omega}^{\alpha}(x)|r|^{q-1} r,
$$

with $\alpha>-2$ and $1<q<(N+1+\alpha) /(N-1)$, it is proved respectively in [13] and [27] that for any $a \in \partial \Omega, u_{\infty, a}$ is a solution of (0.1) in $\Omega$ vanishing on $\partial \Omega \backslash\{a\}$, with a strong singularity at $a$. In those two cases the boundary trace of a nonnegative solution of (0.1) can be any outer regular Borel measure on $\partial \Omega$.

Another interesting type of problems deals with the situation in which the absorption term is strongly degenerate at the boundary. The model example is

$$
g(x, r)=\exp \left(-\kappa / \rho_{\partial \omega}(x)\right) u^{q},
$$

with $q>1$ and $\kappa>0$. In this case the function $g$ belongs to $\mathcal{H} \mathcal{G}_{0}$ for any $q>1$. Therefore fundamental solutions always exist, but a new phenomenon appears which is to be compared with what is called instantaneous or complete blow-up for parabolic equations ([2]) or elliptic equations ([7]), linear or nonlinear, with an inverse square potential.

PROPOSITION 5.1. For any $q>1, \kappa>0$ and $a \in \partial \Omega$

$$
u_{\infty, a}=u_{m}
$$


where $u_{m}$ is the minimal solution of

$$
\begin{aligned}
& -\Delta u+\exp \left(-\kappa / \rho_{\partial \omega}(x)\right) u^{q}=0 \text { in } \Omega, \\
& \lim _{\rho_{\partial \Omega}(x) \rightarrow 0} u(x)=\infty .
\end{aligned}
$$

Proof. Without any loss of generality we assume $a=O$. Let $\left\{x=\left(x_{1}, x^{\prime}\right)\right\}$ be the coordinates in $\mathbb{R}^{N}$. We assume that the hyperplane $H_{0}=\left\{x=\left(0, x^{\prime}\right)\right\}$ is tangent to $\partial \Omega$ at $O$ and $S_{+}^{N-1}=B_{1}(O) \cap\left\{x=\left(x_{1}, x^{\prime}\right): x_{1}>0\right\}$. We write

$$
\exp \left(-\kappa / \rho_{\partial \omega}(x)\right)=h\left(\rho_{\partial \omega}(x)\right) \text {. }
$$

STEP 1 The case $1<q<(N+1) /(N-1)$. For $0<\varepsilon \leq \beta_{0}$, the function $u_{\infty, O}$ is minorized in $\Omega_{\varepsilon}^{m}=\left\{x \in \Omega:|x|<m, 0<\rho_{\partial \omega}(x)<\varepsilon\right\}(m>0$ small enough) by the function

$$
\ell(\varepsilon) U_{\varepsilon}
$$

where $\ell(\varepsilon)=h^{-1 /(q-1)}(\varepsilon)$ and $U_{\varepsilon}$ is the unique solution of

$$
\begin{array}{cl}
-\Delta v=v^{q} & \text { in } \Omega_{\varepsilon}^{m}, \\
v=\infty \delta_{O} & \text { on } \partial \Omega_{\varepsilon}^{m} .
\end{array}
$$

Moreover there holds (see [15])

$$
\lim _{\substack{x \rightarrow O \\ x \in \Omega_{\varepsilon}}}|x|^{2 /(q-1)} U_{\varepsilon}(x)=\omega(x /|x|),
$$

where $\omega$ is the unique positive solution of

$$
\begin{array}{r}
-\Delta_{\sigma} \omega-\left(\frac{2}{q-1}\right)\left(\frac{2 q}{q-1}-N\right) \omega+\omega^{q}=0 \text { in } S_{+}^{N-1}, \\
\omega=0 \text { on } \partial S_{+}^{N-1} .
\end{array}
$$

If we write

$$
U_{\varepsilon}(x)=\varepsilon^{-2 /(q-1)} U_{1, \varepsilon}(x / \varepsilon)=\varepsilon^{-2 /(q-1)} U_{1, \varepsilon}(y), \quad y=x / \varepsilon,
$$

the function $U_{1, \varepsilon}$ satisfies

$$
\Delta U_{1, \varepsilon}=U_{1, \varepsilon}^{q} \quad \text { in } \quad \mathfrak{D}_{\varepsilon}^{m}=\varepsilon^{-1} \Omega_{\varepsilon}^{m}
$$

When $\varepsilon \rightarrow 0, D_{\varepsilon}^{m}$ converges to $\mathfrak{D}_{0}=(0,1) \times \mathbb{R}^{N-1}$ in the sense of sets. Thus, for any $0<\theta_{1}<\theta_{2}<1$, there exists $\varepsilon_{0}$ such that if $0<\varepsilon \leq \varepsilon_{0}$, the following inclusion holds

$$
\mathfrak{G}_{\theta}^{m}=\left\{y=\left(y_{1}, y^{\prime}\right): \theta_{1}<y_{1}<\theta_{2}, 1<\left|y^{\prime}\right| \leq m / 2 \varepsilon\right\} \subset \mathfrak{D}_{\varepsilon}^{m} .
$$


Because

$$
U_{1, \varepsilon}(y) \leq C|y|^{-2 /(q-1)},
$$

$U_{1, \varepsilon}$ converges uniformly, as $\varepsilon \rightarrow 0$, on any compact subset of $\mathfrak{D}_{0} \backslash\{O\}$ to the unique solution $U_{1}$ of

$$
\Delta U_{1}=U_{1}^{q} \quad \text { in } \quad \mathfrak{D}_{0}
$$

which vanishes on $\partial \mathfrak{D}_{0} \backslash\{O\}$ and satisfies

$$
\lim _{\substack{y \rightarrow O \\ y \in \mathfrak{D}_{0}}}|y|^{2 /(q-1)} U_{1}(y)=\omega(y /|y|) .
$$

Therefore there exists some $\eta \in(0,1)$ such that

$$
U_{1, \varepsilon}\left(y_{1}, y^{\prime}\right) \geq \eta \sin \left(\frac{\pi\left(y_{1}-\theta_{1}\right)}{\theta_{2}-\theta_{1}}\right), \quad \text { for } y_{1} \in\left[\theta_{1}, \theta_{2}\right] \text { and }\left|y^{\prime}\right|=1 \text {. }
$$

Notice that the function $y_{1} \mapsto \psi_{\theta}\left(y_{1}\right)=\sin \left(\pi\left(y_{1}-\theta_{1}\right) /\left(\theta_{2}-\theta_{1}\right)\right)$ vanishes for $y_{1}=\theta_{1}$ and for $y_{1}=\theta_{2}$.

We first suppose $N=2$. Then for any $\beta>0$ the function

$$
y^{\prime} \mapsto \varphi_{\beta}\left(y^{\prime}\right)=\frac{\sinh \left(\beta\left(\frac{m}{2 \varepsilon}-\left|y^{\prime}\right|\right)\right)}{\sinh \left(\beta\left(\frac{m}{2 \varepsilon}-1\right)\right)},
$$

is nonnegative takes the value 1 for $\left|y^{\prime}\right|=1$, and vanishes for $\left|y^{\prime}\right|=m /(2 \varepsilon)$. If we set

$$
\zeta_{\theta, \beta}\left(y_{1}, y^{\prime}\right)=\eta \psi_{\theta}\left(y_{1}\right) \varphi_{\beta}\left(y^{\prime}\right)
$$

there holds

$$
\Delta \zeta_{\theta, \beta}=\left(\beta^{2}-\frac{\pi^{2}}{\left(\theta_{2}-\theta_{1}\right)^{2}}\right) \zeta_{\theta, \beta}
$$

Since $\zeta_{\theta, \beta} \leq \eta$, it follows

$$
\Delta \zeta_{\theta, \beta} \geq\left(\beta^{2}-\frac{\pi^{2}}{\left(\theta_{2}-\theta_{1}\right)^{2}}\right) \eta^{1-q} \zeta_{\theta, \beta}^{q} \quad \text { in } \quad \mathfrak{G}_{\theta}^{m},
$$

furthermore

$$
\begin{array}{ll}
\zeta_{\theta, \beta}\left(y_{1}, y^{\prime}\right)=0 & \text { for } y_{1}=\theta_{i}, i=1,2 \\
\zeta_{\theta, \beta}\left(y_{1}, y^{\prime}\right)=0 & \text { for } \quad\left|y^{\prime}\right|=m / 2 \varepsilon \\
\zeta_{\theta, \beta}\left(y_{1}, y^{\prime}\right)=0 & \text { for } \quad\left|y^{\prime}\right|=1
\end{array}
$$


We chose $\beta$ such that $\frac{\pi^{2}}{\left(\theta_{2}-\theta_{1}\right)^{2}} \eta^{1-q}=1$. By (5.11) and the maximum principle one obtains

$$
U_{1, \varepsilon}\left(y_{1}, y^{\prime}\right) \geq \zeta_{\theta, \beta}\left(y_{1}, y^{\prime}\right) \quad \text { in } \mathfrak{G}_{\theta}^{m}
$$

Therefore

$$
\begin{aligned}
u\left(x_{1}, x^{\prime}\right) & \geq \ell(\varepsilon) \varepsilon^{-2 /(q-1)} U_{1, \varepsilon}\left(x_{1} / \varepsilon, x^{\prime} / \varepsilon\right) \\
& \geq \ell(\varepsilon) \varepsilon^{-2 /(q-1)} \zeta_{\theta, \beta}\left(x_{1} / \varepsilon, x^{\prime} / \varepsilon\right),
\end{aligned}
$$

for $\theta_{1} \varepsilon \leq x_{1} \leq \theta_{1} \varepsilon$ and $\varepsilon \leq\left|x^{\prime}\right| \leq m / 2$. Take $x_{1}=\varepsilon\left(\theta_{1}+\theta_{2}\right) / 2=\theta \varepsilon$, then

$$
u\left(\theta \varepsilon, x^{\prime}\right) \geq \eta \ell(\varepsilon) \varepsilon^{-2 /(q-1)} \frac{\sinh \left(\beta\left(\frac{m-2\left|x^{\prime}\right|}{2 \varepsilon}\right)\right)}{\sinh \left(\beta\left(\frac{m}{2 \varepsilon}-1\right)\right)} .
$$

If $|x|^{\prime} \leq m / 4$

$$
\frac{\sinh \left(\beta\left(\frac{m-2\left|x^{\prime}\right|}{2 \varepsilon}\right)\right)}{\sinh \left(\beta\left(\frac{m}{2 \varepsilon}-1\right)\right)}=e^{\beta\left(1-|x|^{\prime} / \varepsilon\right)}(1+\circ(1)) \text { as } \varepsilon \rightarrow 0,
$$

Thus

$$
u\left(\theta \varepsilon, x^{\prime}\right) \geq \eta \ell(\varepsilon) \varepsilon^{-2 /(q-1)} e^{\beta} e^{-\beta\left|x^{\prime}\right| / \varepsilon}(1+\circ(1))
$$

and

$$
\liminf _{\varepsilon \rightarrow 0} u\left(\theta \varepsilon, x^{\prime}\right) \geq \eta e^{\beta} \liminf _{\varepsilon \rightarrow 0} \ell(\varepsilon) \varepsilon^{-2 /(q-1)} e^{-\beta\left|x^{\prime}\right| / \varepsilon} .
$$

Since $\ell(\varepsilon)=e^{\kappa /((q-1) \varepsilon}$,

$$
\ell(\varepsilon) \varepsilon^{-2 /(q-1)} e^{\beta\left(1-|x|^{\prime} / \varepsilon\right)} \geq \varepsilon^{-2 /(q-1)} \exp \left[\varepsilon^{-1}\left(\kappa /(q-1)-\beta|x|^{\prime}\right)\right]
$$

If we fix $|x|^{\prime}<\beta \kappa /(q-1)$, then

$$
\liminf _{\varepsilon \rightarrow 0} u\left(\theta \varepsilon, x^{\prime}\right)=\lim _{\rho_{\partial \Omega}(x) \rightarrow 0} u(x)=\infty,
$$

and this limit is uniform on any compact subset of $\left\{x^{\prime}:|x|^{\prime}<\beta \kappa /(q-1)\right\}$, which is equivalent to any compact of $\{x:|\sigma(x)|<\beta \kappa /(q-1)\}$. Put $\tau=$ $\beta \kappa /(2(q-1))$. Because this blow-up holds in a fixed neighborhood $\partial \Omega \cap \bar{B}_{\tau}(O)$ of $O$, we can replace $O$ by any point $P$ in $\partial \Omega \cap \bar{B}_{\tau}(O)$ and conclude that

$$
\lim _{\rho_{\partial \Omega}(x) \rightarrow 0} u(x)=\infty,
$$


uniformly if $|\sigma(x)-P| \leq \tau$. Iterating this process infers that

$$
\lim _{\rho_{\partial \Omega}(x) \rightarrow 0} u(x)=\infty .
$$

Next we assume $N \geq 3$. Let $\beta>0$ to be fixed and

$$
\Gamma_{\varepsilon}=\left\{y^{\prime} \in \mathbb{R}^{N-1}: 1<\left|y^{\prime}\right|<m / 2 \varepsilon\right\}
$$

and let $B_{\beta}\left(y^{\prime}\right)$ be the solution of

$$
\begin{aligned}
& \Delta_{y^{\prime}} B_{\beta}=\beta^{2} B_{\beta} \quad \text { in } \Gamma_{\varepsilon}, \\
& B_{\beta}\left(y^{\prime}\right)=1 \quad \text { if } \quad\left|y^{\prime}\right|=1, \\
& B_{\beta}\left(y^{\prime}\right)=0 \text { if }\left|y^{\prime}\right|=m / 2 \varepsilon .
\end{aligned}
$$

The function $\zeta_{\theta, \beta}\left(y_{1}, y^{\prime}\right)=\eta \psi_{\theta}\left(y_{1}\right) B_{\beta}\left(y^{\prime}\right)$ satisfies also (5.13) in $\mathfrak{G}_{\theta}^{m}$. Therefore, if we chose $\beta$ as in the case $N=2,(5.15)$ is still valid. Since $B_{\beta}$ is a Bessel function, its behaviour at infinity is classical and there holds, for $|x|^{\prime} \leq m / 4$,

$$
B_{\beta}(y)=C_{\beta}\left(\frac{|x|^{\prime}}{\varepsilon}\right)^{1-N / 2} e^{-\beta|x|^{\prime} / \varepsilon}(1+\circ(1)), \quad \text { as } \varepsilon \rightarrow 0
$$

We conclude as in the case $N=2$.

SteP 2. The general case. If $q \geq(N+1) /(N-1)$ let $\alpha>0$ such that

$$
q<(N+1+\alpha) /(N-1)
$$

We write

$$
h\left(\rho_{\partial \Omega}(x)\right)=\rho_{\partial \Omega}^{\alpha}(x) \tilde{h}\left(\rho_{\partial \Omega}(x)\right)
$$

with

$$
\tilde{h}\left(\rho_{\partial \Omega}(x)\right)=\rho_{\partial \Omega}^{-\alpha}(x) h\left(\rho_{\partial \Omega}(x)\right) .
$$

We can assume that $r \mapsto \tilde{h}(r)$ is nondecreasing near $r=0$ and we extend it by continuity at $r=0$ by putting $\tilde{h}(0)=0$. Thus there holds

$$
\Delta u \leq \tilde{h}(\varepsilon) \rho_{\partial \Omega}^{\alpha}(x) u^{q} \quad \text { in } \Omega_{\varepsilon} .
$$

The equation

$$
-\Delta U+\rho_{\partial \Omega}^{\alpha}(x) U^{q}=0,
$$

admits weak and strong isolated singularities on the boundary and any positive solution with a strong singularity at $x=O$ satisfies

$$
\lim _{\substack{x \rightarrow O \\ x \in \Omega_{\varepsilon}}}|x|^{(2+\alpha) /(q-1)} U_{\varepsilon}(x)=\omega_{\alpha}(x /|x|),
$$


where $\omega_{\alpha}$ is the unique positive solution of

$$
\begin{array}{r}
-\Delta_{\sigma} \omega_{\alpha}-\left(\frac{2+\alpha}{q-1}\right)\left(\frac{2 q+\alpha}{q-1}-N\right) \omega_{\alpha}+\omega_{\alpha}^{q}=0 \text { in } S_{+}^{N-1}, \\
\omega=0 \text { on } \partial S_{+}^{N-1},
\end{array}
$$

we proceed as in Step 1, with some minor changes of coefficients.

STEP 3. End of the proof. The minimal solution $u_{m}$ of (5.6) is constructed by considering the increasing sequence $u_{k}$ of solutions of

$$
\begin{aligned}
-\Delta u_{k}+\exp \left(-\kappa / \rho_{\partial \omega}(x)\right) u_{k}^{q}=0 & \text { in } \Omega, \\
u_{k}(x)=k & \text { on } \partial \Omega .
\end{aligned}
$$

When $k \rightarrow \infty, u_{k} \rightarrow u_{m}$, thus $u_{\infty, a} \geq u_{m}$. On the other hand, $u_{\lambda \delta_{a}}$ is constructed by approximating the Dirac mass on the boundary by bounded functions $g_{\lambda}$. Thus the corresponding solutions $u_{g_{\lambda}}$ of $(0.4)$ are all dominated by $u_{m}$. Therefore

$$
u_{\lambda \delta_{a}} \leq u_{m} \Longrightarrow u_{\infty, a} \leq u_{m} .
$$

REMARK 5.2. If the domain $\Omega$ is starshaped with respect to some point, say $O$, the Iscoe uniqueness method (see [17]) of scaling applies straightforwardly to prove the uniqueness of the solution of (5.6). We recall this method. Let $\ell>0$ and $u_{\ell}(x)=\ell^{2 /(q-1)} u(\ell x)$, then $u_{\ell}$ satisfies

$$
\begin{aligned}
-\Delta u_{\ell}+e^{-\kappa / \rho_{\partial \Omega}(\ell x)} u_{\ell}^{q} & =0 \quad \text { in } \quad \Omega_{\ell}=\frac{1}{\ell} \Omega, \\
u_{\ell} & =\infty \quad \text { on } \partial \Omega_{\ell} .
\end{aligned}
$$

But $\Omega_{\ell} \subset \Omega$ and $e^{-\kappa / \rho_{\partial \Omega}(\ell x)} \leq e^{-\kappa / \rho_{\partial \Omega}(x)}$ if $\ell>1$. Therefore $u_{\ell}$ satisfies

$$
-\Delta u_{\ell}+e^{-\kappa / \rho_{\partial \Omega}(x)} u_{\ell}^{q} \geq 0, \quad \text { in } \Omega_{\ell} .
$$

If $\hat{u}$ is another of (5.6) in $\Omega$, then $u_{\ell} \geq \hat{u}$. Letting $\ell \rightarrow 1$ infers $u \geq \hat{u}$. In the same way $\hat{u} \geq u$. In a much more elaborated manner, if $\Omega$ is locally a continuous graph, the method of local translations developped by the authors in [21] can be adapted and once again uniqueness of the solution of (5.6) holds.

Combining Proposition 5.1, the previous remark and Theorem 4.1 (in the case $1<q<(N+1) /(N-1))$ we derive,

COROLlary 5.2. Let $q>1$ and $u$ be a nonnegative solution of

$$
-\Delta u+\exp \left(-1 / \rho_{\partial \omega}(x)\right) u^{q}=0 \quad \text { in } \Omega .
$$

Then either

(i) $\mathcal{S}(u)=\partial \Omega, \operatorname{Tr}_{\partial \Omega}^{e}(u)$ the Borel measure indentically equal to $\infty$, and

$$
u_{\infty, a}=u_{m}
$$

or

(ii) $\mathcal{R}(u)=\partial \Omega$ and $\operatorname{Tr}_{\partial \Omega}^{e}(u)=v$ is a bounded Borel measure. Moreover, if $1<q<(N+1) /(N-1)$, $v$ is a Radon measure and $u=u_{v}$. 


\section{REFERENCES}

[1] P. Baras - M. Pierre, Singularités éliminables pour des équations semi-linéaires, Ann. Inst. Fourier (Grenoble) 34 (1984), 185-206.

[2] P. BARAs - J. GoldSTEIn, The heat equation with a singular potential, Trans. Amer. Math. Soc. 284 (1984), 121-139.

[3] Рh. Benilan - H. Brezis, Nonlinear problems related to the Thomas-Fermi equation, unpublished paper, see [6].

[4] M. F. Bidaut-VéRon - L. Vivier, An elliptic semilinear equation with source term involving boundary measures: the subcritical case, Rev. Mat. Iberoamericana 16 (2000), 477-513.

[5] H. BrezIs, Une équation semi-linéaire avec conditions aux limites dans $L^{1}$, unpublished paper. See also [32]-Chap. 4.

[6] H. BREZIS, Some variational problems of the Thomas-Fermi type, in "Variational Inequalities”, R. W. Cottle, F. Giannessi and J.-L. Lions (eds.), Wiley, Chichester (1980), 53-73.

[7] X. CABRE, Extremal solutions and instantaneous complete blow-up for elliptic and parabolic problems, preprint.

[8] R. Dautray - J. L. Lions, "Analyse Mathématique et Calcul Numérique”, Masson, Paris, 1987.

[9] J. DooB, "Classical Potential Theory and its Probabilistic Counterpart", Springer-Verlag, Berlin-New York, 1984.

[10] E. B. Dynkin - S. E. Kuznetsov, Trace on the boundary for solutions of nonlinear differential equations, Trans. Amer. Math. Soc. 350 (1998), 4499-4519.

[11] E. B. Dynkin - S. E. Kuznetsov, Solutions of nonlinear differential equtions on a Riemannian manifold and their trace on the Martin boundary, Trans. Amer. Math. Soc. 350 (1998), 4521-4552.

[12] E. B. DynKin - S. E. KuzNETsov, Fine topology and fine trace on the boundary associated with a class of quasilinear differential equations, Comm. Pure Appl. Math. 51 (1998), 897936.

[13] J. FABBRI - J. R. LiCOIS, Behavior at boundary of solutions of a weakly superlinear elliptic equation, Adv. Nonlinear Stud. 2 (2002), 147-176.

[14] D. Gilbarg - N. S. Trudinger, "Partial Differential Equations of Second Order", 2nd Ed. Springer-Verlag, Berlin-New York, 1983.

[15] A. GMira - L. VÉRon, Boundary singularities of solutions of some nonlinear elliptic equations, Duke Math. J. 64 (1991), 271-324.

[16] M. GRILlot - L. VÉRON, Boundary trace of solutions of the Prescribed Gaussian curvature equation, Proc. Roy. Soc. Edinburgh 130 A (2000), 1-34.

[17] I. IsCOE, On the support of measure-valued critical branching Brownian motion, Ann. Prob. 16 (1988), 200-221.

[18] J. B. Keller, On solutions of $\Delta u=f(u)$, Comm. Pure Appl. Math. 10 (1957), 503-510.

[19] J. F. Le Gall, Les solutions positives de $\Delta u=u^{2}$ dans le disque unité, C.R. Acad. Sci. Paris 317 Ser. I (1993), 873-878.

[20] J. F. LE GALL, The brownian snake and solutions of $\Delta u=u^{2}$ in a domain, Probab. Theory Related Fields 102 (1995), 393-432. 
[21] M. MARCUS - L. VÉRON, Uniqueness and asymptotic behaviour of solutions with boundary blow-up for a class of nonlinear elliptic equations, Ann. Inst. H. Poincaré 14 (1997), 237274.

[22] M. MARCUS - L. VÉRON, Traces au bord des solutions positives d'équations elliptiques non-linéaires, C.R. Acad. Sci. Paris 321 Ser. I (1995), 179-184.

[23] M. MARCus - L. VÉron, Traces au bord des solutions positives d'équations elliptiques et paraboliques non-linéaires: résultats d'existence et d'unicité, C.R. Acad. Sci. Paris $\mathbf{3 2 3}$ Ser. I (1996), 603-608.

[24] M. MARCUS - L. VÉRon, The boundary trace of positive solutions of semilinear elliptic equations: the subcritical case, Arch. Ration. Mech. Anal. 144 (1998), 201-231.

[25] M. MARCUS - L. VÉRON, The boundary trace of positive solutions of semilinear elliptic equations: the supercritical case, J. Math. Pures Appl. 77, 481-524 (1998).

[26] M. Marcus - L. Véron, Removable singularities and boundary traces, J. Math. Pures Appl. 80 (2001), 879-900.

[27] M. MARCUS - L. VÉRON, The boundary trace and generalyzed boundary value problem for semilinear elliptic equations with coercive absorption, Comm. Pure Appl. Math. 56 (2003), 0689-0731.

[28] M. MARCUS - L. VÉRON, Initial trace of positive solutions to semilinear parabolic inequalities, Adv. Nonlinear Studies 2 (2002), 395-436.

[29] A. RAtTo - M. Rigoli - L. VÉRON, Scalar curvature and conformal deformation of hyperbolic space, J. Funct. Anal. 121 (1994), 15-77.

[30] Y. RichARD - L. VÉRON, Isotropic singularities of nonlinear elliptic inequalities, Ann. Inst. H. Poincaré 6 (1989), 37-72.

[31] J. L. VAZQUEZ, An a priori interior estimate for the solution of a nonlinear problem representing weak diffusion, Nonlinear Anal. 5 (1981), 119-135.

[32] L. VÉRON, "Singularities of Solutions of Second Order Quasilinear Equations", Pitman Research Notes in Math. 353, Addison Wesley Longman Inc., 1996.

Department of Mathematics, Israel Institute of Technology Technion, Haifa 32000, Israel marcusm@math.technion.ac.il

Laboratoire de Mathématiques et Physique Théorique CNRS UMR 6083 Université François Rabelais Tours 37200, France veronl@univ-tours.fr 